\title{
iMLCA: Machine Learning-powered Iterative Combinatorial Auctions with Interval Bidding*
}

\author{
Benjamin Lubin ${ }^{\dagger}$ \\ Boston University \\ blubin@bu.edu
}

\author{
Sven Seuken ${ }^{\dagger}$ \\ University of Zurich \\ seuken@ifi.uzh.ch
}

\author{
Manuel Beyeler ${ }^{\dagger}$ \\ University of Zurich \\ manuel.beyeler@uzh.ch
}

\author{
Gianluca Brero \\ Harvard University \\ gbrero@g.harvard.edu
}

Preference elicitation is a major challenge in large combinatorial auctions because the bundle space grows exponentially in the number of items. Recent work has used machine learning (ML) algorithms to identify a small set of bundles to query from each bidder. However, a shortcoming of this prior work is that bidders must submit exact values for the queried bundles, which can be quite costly. To address this, we propose $i M L C A$, a new ML-powered iterative combinatorial auction with interval bidding (i.e., where bidders submit upper and lower bounds instead of exact values). To steer the auction towards an efficient allocation, we introduce a price-based activity rule, asking bidders to tighten bounds on relevant bundles only. In our experiments, iMLCA achieves the same allocative efficiency as the prior ML-based auction that uses exact bidding. Moreover, it outperforms the well-known combinatorial clock auction in a realistically-sized domain.

Key words: Combinatorial Auction, Machine Learning, Interval Bidding

\section{Introduction}

Combinatorial auctions (CAs) are used to allocate a set of heterogeneous items to multiple bidders who may see the items as substitutes or complements. In CAs, bidders can submit bid on bundles rather than just individual items, which avoids the exposure problem. CAs are widely used in practice, e.g., for procurement (Sandholm 2013) and for TV-ad allocation (Goetzendorf et al. 2015).

Unfortunately, in CAs, the bundle space grows exponentially in the number of items, which makes it impractical for bidders to explore and report their full value function, even in medium-

* Some of the ideas presented in this paper were also described in a one-page abstract that was published in the conference proceedings of the 22nd ACM Conference on Electronic Commerce (EC'21) (Beyeler et al. 2021).

$\dagger$ These authors have contributed equally to this paper. 
sized domains. Therefore, iterative combinatorial auctions (ICAs) are often used in practice, where the auctioneer interacts with the bidders over the course of multiple rounds and only elicits a limited amount of information. A prime example is the combinatorial clock auction (CCA), which has already been used for more than 15 auctions and has generated more than $\$ 15$ Billion since 2008 (Ausubel and Baranov 2017) in applications such as spectrum auctions (Cramton 2013) and auctioning off the right to build offshore wind farms (Ausubel and Cramton 2011).

To be practical, the CCA uses simple (linear) per-item prices in the first phase of the auction, and it limits the number of bids that can be submitted in the second phase. For example, in the 2014 Canadian spectrum auction (with 98 goods), bidders could only submit up to 500 bids, which is a tiny fraction of the whole bundle space. But these simplifications are not without loss. As Scheffel et al. (2012) and Bichler et al. (2013) have shown, bidders tend to focus on only a small number of bundles too early in the auction, which can lead to large efficiency losses.

\subsection{ML-powered ICAs with Exact Bidding}

To address these problems with the CCA, Brero et al. $(2018,2021 b)$ introduced MLCA, a machine learning (ML)-powered iterative combinatorial auction. MLCA drives the elicitation process via an ML-powered query module (instead of using prices). This query module learns bidders' value functions to identify which value queries to ask in each round. Importantly, Brero et al. (2021b) have shown that MLCA outperforms the CCA in terms of efficiency in a large CA domain.

A shortcoming of MLCA is that it requires bidders to answer each query with an exact value. In many applications, this is difficult for bidders, because determining the exact value of even a single bundle typically involves a complex decision-making problem (Parkes 2006). For example, in spectrum auctions, a bundle of licenses corresponds to a mobile network operator being able to execute on a business plan (like offering a national cell plan). Thus, determining the value of a bundle of licenses is a multi-year profit-optimization problem, which requires time and resources to solve. Furthermore, submitting a bid close to a bidder's true value for a bundle (i.e., his maximum willingness-to-pay) often requires board approval, which involves a costly process (Bichler and 
Paulsen 2018). In some CAs (e.g., for oil drilling rights), it may even be practically impossible to determine exact values, because there is inherent uncertainty about the value of the resources that cannot be resolved before the auction (Oren and Williams 1975). Requiring exact values is also costly from a computational perspective. While large-scale profit-optimization problems can be solved via mixed integer programming, this is a strongly NP-hard problem (Garey and Johnson 1978); thus, no FPTAS exists. However, anytime primal-dual algorithms that output upper and lower bounds exist. Thus, computing bounds is easier than computing exact values.

\subsection{Our Contribution: iMLCA}

To address the difficulty of reporting exact values, we propose $i M L C A$, a new ML-powered combinatorial auction with interval bidding. iMLCA asks bidders to report upper and lower bounds on their values (i.e., intervals). For our design, we take the MLCA mechanism by Brero et al. (2021b) as the starting point but we extensively modify and extend it to handle the new interval queries. While interval bidding simplifies the interaction with the auction, we must take great care to still achieve high efficiency. Therefore, a significant aspect of our contribution is to combine an ML-based approach with interval-based bidding into a single coherent design.

The main challenge is that bidders' reported intervals will often be overlapping, such that one cannot determine the efficient allocation nor reasonable payments. Consequently, iMLCA must force bidders to successively tighten their reported intervals, but without requiring bounds to be tightened so much that the benefits of interval bidding are obviated. To this end, we design a price-based activity rule, extending earlier work on refinement processes by Lubin et al. (2008), which makes for an easily-understood bidder interaction. Using prices in the activity rule implies that quoting the right prices is of utmost importance, such that bound tightening only happens where needed. Accordingly, one of our technical contributions is a new algorithm for generating approximate linear clearing prices. ${ }^{1}$ We carefully integrate our new price-based activity rule with

\footnotetext{
${ }^{1}$ Note that we only use linear prices to drive the bound refinement process, while the elicitation (i.e., deciding which queries to ask) is driven by a sophisticated ML algorithm. This is in contrast to the CCA, where linear prices are used to drive the elicitation process.
} 
the ML-powered query module designed by Brero et al. (2021b), such that they can be executed in the same step in each auction round (phase 2 of iMLCA; see Section 4.2).

To ensure that the bounds are eventually tight enough to determine the efficient allocation (given reports), we design an additional Convergence Phase (phase 3 of iMLCA; see Section 4.3). In this phase, iMLCA asks bidders to tighten their bounds on a few bundles (four in our implementation), until a certain convergence criterion is met. By setting this criterion, the auctioneer can decide on a trade-off between mitigating bidders' incentives to manipulate and bidders' elicitation efforts. Ultimately, the final allocation and prices are determined at the lower bounds only (Section 4.4).

In Section 5, we study the theoretical properties of iMLCA. Given our design, it is straightforward to prove that iMLCA satisfies individual rationality and no-deficit. In contrast, the incentive analysis requires more care. While iMLCA (like the CCA and MLCA) is not strategyproof, we explain how our design choices lead to good incentives in practice for iMLCA. Finally, we prove that our new price generation algorithm leads to fewer bound refinements than earlier algorithms.

In Section 6, we provide a detailed experimental evaluation of iMLCA using the spectrum auction test suite (SATS) (Weiss et al. 2017). We show that iMLCA achieves the same efficiency as MLCA, but with much lower elicitation cost. Furthermore, we show that iMLCA even outperforms the CCA in terms of efficiency in a realistically-sized CA domain. Regarding incentives, we provide experimental evidence showing that bidders cannot benefit by misreporting their bounds. Finally, in Section 7, we discuss limitations of our approach as well as potential extensions.

\section{Related Work}

Our work lies in the broad area of research on preference elicitation algorithms (see Sandholm and Boutilier (2006) for a survey). Lahaie and Parkes (2004) and Blum et al. (2004) were the first to use ML to improve preference elicitation. They showed that for certain classes of valuations, ML can be used to design tractable elicitation algorithms.

The work by Brero and Lahaie (2018) and Brero et al. (2019) follows a design paradigm that is similar to ours (i.e., integrating an ML algorithm into the auction itself). They design a Bayesian 
price-based mechanism, where the main goal is to improve the speed of an iterative CA until it converges to clearing prices. Similarly, Shen et al. (2019) also used ML to learn clearing prices, but in an ad auction context instead of an iterative CA setting. In contrast to these works, we do not aim to directly learn clearing prices (which may not exist in our setting); instead, we use ML to learn the bidders' value functions, and we use this to steer the iterative query process.

Recently, multiple variations of MLCA have been proposed. For example, Weissteiner and Seuken (2020) showed how to use neural networks (NNs) as the ML algorithm in MLCA in place of support vector regression (SVR), which can further increase efficiency in some domains. Similarly, Weissteiner et al. (2021) showed how using Fourier Analysis as part of an MLCA-based design can be useful. In contrast to iMLCA, these papers also require bidders to report exact values.

There is also related work using ML for automated mechanism design (Dütting et al. 2015, 2019, Golowich et al. 2018). This work has focused on learning allocation and payment rules, so that the resulting mechanism achieves high efficiency or revenue and is (approximately) truthful. Recently, Brero et al. (2021a) used reinforcement learning to learn optimal indirect mechanisms within the restricted class of sequential price mechanisms. All of these works use ML to learn the mechanism; but in contrast to iMLCA, the final (learned) mechanism does not use ML when executed.

Bünz et al. (2018) studied the automated search for good core-selecting mechanisms. The authors incorporated an equilibrium solver into their search algorithm, such that they could algorithmically decide how close to strategyproof different mechanisms are. Unfortunately, their approach does not yet scale to large settings nor to iterative auction design problems. Tang (2017) proposed a similar approach for optimizing mechanisms in dynamic environments like ad auctions. Instead of employing an equilibrium solver, they use ML to model agents' behavior inside the optimization.

\section{Preliminaries}

In this section, we present our formal model and provide a summary of the MLCA mechanism.

\subsection{Formal Model}

CAs allocate $m$ indivisible items among $n$ bidders. We let $M=\{1, \ldots, m\}$ denote the set of items and $N=\{1, \ldots, n\}$ the set of bidders. Bundles of items are represented by an indicator vector 
$x \in \mathcal{X}=\{0,1\}^{m}$, i.e., $x_{j}=1$ iff item $j \in M$ is contained in bundle $x$. Each bidder $i$ 's preferences are captured by a value function denoted $v_{i}: \mathcal{X} \rightarrow \mathbb{R}_{\geq 0}$. We let $v=\left(v_{1}, \ldots, v_{n}\right)$ denote the value profile of all bidders. Without loss of generality, we assume that the value functions are normalized such that the value of the empty bundle is zero; importantly, we impose no further structural restrictions.

We also refer to the set of all bidders $N$ as the main economy. For this economy, an allocation is denoted by $a=\left(a_{1}, \ldots, a_{n}\right) \in \mathcal{X}^{n}$, where $a_{i}$ is the bundle allocated to bidder $i$. We denote the set of feasible allocations by $\mathcal{F}=\left\{a \in \mathcal{X}^{n}: \sum_{i \in N} a_{i j} \leq 1, \forall j \in M\right\}$. Sometimes, we need to work with a marginal economy, where a single bidder $i$ is omitted. In this case, we denote the allocation in the marginal economy by $a^{-i}=\left(a_{1}^{-i}, \ldots, a_{i-1}^{-i}, a_{i+1}^{-i}, \ldots, a_{n}^{-i}\right)$, where the superscript " $-i$ " indicates which bidder has been excluded.

A bidder $i$ may make non-truthful value reports $\hat{v}_{i}$ to the mechanism, giving rising to value profiles other than the true one. For any profile $\hat{v}$, we let $\hat{v}(a)=\sum_{i} \hat{v}_{i}\left(a_{i}\right)$ denote the total reported value of allocation $a$. When the true value profile is used, this quantity is called the social welfare of the allocation. An allocation that maximizes the social welfare is denoted $a^{*} \in \arg \max _{a \in \mathcal{F}} v(a)$. We let $p_{i}$ denote the payment charged to bidder $i$, and we let $p=\left(p_{1}, \ldots, p_{n}\right)$ denote the payment profile for all bidders. We assume quasi-linear utilities of the form $u_{i}(a, p)=v_{i}\left(a_{i}\right)-p_{i}$.

For iMLCA, we introduce an interval query as a generalization of a value query, but where reports specify upper and lower bounds rather than exact values. ${ }^{2}$ We denote the $k^{\text {th }}$ bundle-value report of bidder $i$ by $\left(x_{i k}, \underline{v}_{i k}, \bar{v}_{i k}\right)$, where $\underline{v}_{i k}$ is the lower bound and $\bar{v}_{i k}$ the upper bound reported for bundle $x_{i k}$. Note that we do not use the standard $\hat{v}$ notation here to simplify notation and because intervals are always reports so there is no opportunity for confusion. We denote the set of all bundle-value reports of bidder $i$ as $R_{i}$; the profile of all reports is $R=\left(R_{1}, \ldots, R_{n}\right)$. To simplify notation, we say that $x \in R_{i}$ if there exists $k:\left(x_{i k}, \underline{v}_{i k}, \bar{v}_{i k}\right) \in R_{i}$ and $x_{i k}=x$. For convenience and by slightly overloading notation, we denote by $\underline{v}_{i}(\cdot)$ and $\bar{v}_{i}(\cdot)$ the upper and lower reported value functions for

\footnotetext{
${ }^{2}$ Our interval query is similar to that in the tree-based bidding language (Cavallo et al. 2005) but simpler, in that
} bidders need to specify bounds on only a single bundle rather than a concisely represented set of bundles. 

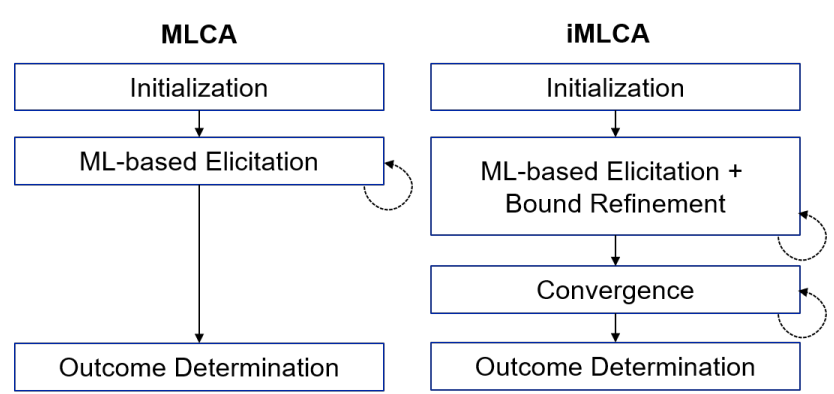

Figure 1 Auction phases of MLCA and iMLCA

each bidder $i$ respectively; note that these functions have support on $R_{i}$ only and are elsewhere undefined. We denote the set of feasible allocations based on reports $R$ as $\mathcal{F}_{R}=\left\{a \in \mathcal{F}: a_{i} \in R_{i} \forall i\right\}$.

We will also need the following valuation function:

Definition 1 (Perturbed Valuation (Lubin et al. 2008)). Bidder i's perturbed valuation function with respect to a given allocation $a$, defined over bundles $x \in R_{i}$, is given by:

$$
\stackrel{\circ}{v}_{i}(x \mid a)= \begin{cases}\underline{v}_{i}(x) & \text { if } x=a_{i} \\ \bar{v}_{i}(x) & \text { otherwise }\end{cases}
$$

In words, the perturbed valuation enables us to capture the worst case loss from allocating bundle $a_{i}$ to bidder $i$ instead of any other bundle $x$. We write $\stackrel{\circ}{v}_{i}(x \mid a)$ as $\stackrel{\circ}{v}_{i}(x)$ where $a$ is clear from context.

We use linear prices in our refinement processes, which we denote by $\pi \in \mathbb{R}_{\geq 0}^{m}$. Clearing prices are prices such that demand meets supply, yielding a feasible allocation $a$ with $v_{i}\left(a_{i}\right)-\pi\left(a_{i}\right) \geq$ $v_{i}(x)-\pi(x) \forall i \in N, x \in \mathcal{X}$ (demand) and $\pi(a) \geq \pi\left(a^{\prime}\right) \forall a^{\prime} \in \mathcal{F}$ (supply). Together, the clearing prices and such an allocation form a competitive equilibrium.

\subsection{MLCA}

The $M L$-powered ICA (MLCA) by Brero et al. (2021b) has the three phases shown in Figure 1:

1. The initialization phase, where random bundles are queried generating input for the ML.

2. The iterative ML-based elicitation phase, where an ML-powered query module is used to generate new queries every round.

3. The outcome determination phase, where the outcome (final allocation and payments) is computed based solely on reported bundle-value pairs. 


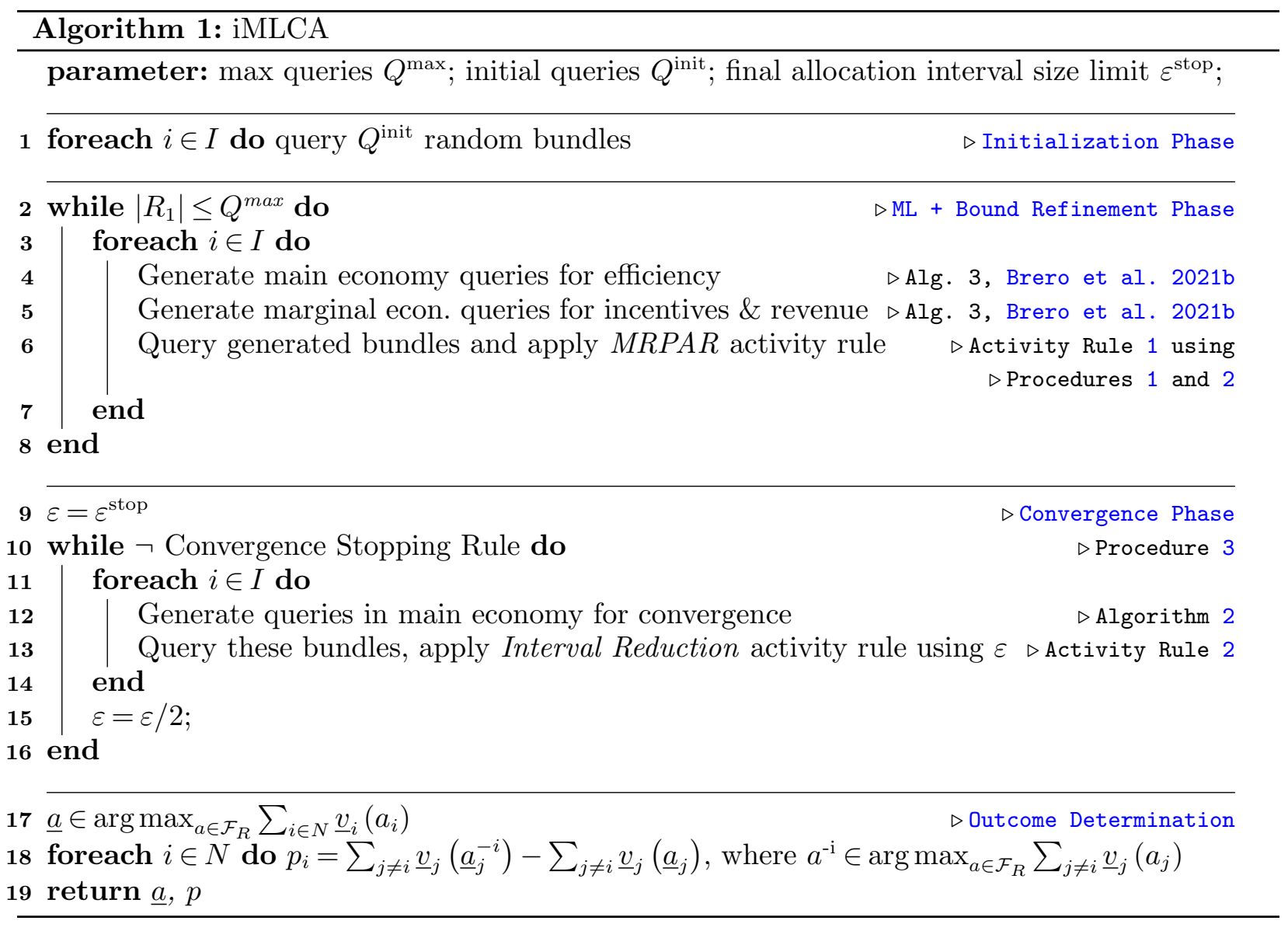

The core of MLCA is the $M L$-powered query module used in phase 2, which, in every round of the auction, aims to generate a query profile that forms a feasible allocation; such bundles will likely be useful when computing the final allocation. Concretely, the query module uses an ML algorithm to generalize from the bundle-value pairs that have already been reported to predict values for all unreported bundles. It then computes the efficient allocation at these learned valuations and queries bidders for their value at this allocation. If the ML algorithm is sufficiently accurate, then the queried allocation will be highly efficient; otherwise, the bidder can correct the ML via the issued query. This way, MLCA proceeds towards a more efficient outcome, assuming a sufficiently effective ML algorithm and truthful bidding. For details, we refer the reader to Brero et al. (2021b).

\section{ML-powered Combinatorial Auction with Interval Bidding}

In this section, we introduce our new ML-powered ICA with interval bidding (iMLCA). From a design perspective, iMLCA builds upon the MLCA design and shares some common components; 
but to facilitate interval bidding, significant changes are necessary. Figure 1 depicts the differences between the mechanisms. Specifically, iMLCA enhances the ML phase by adding a bound refinement component that requires bidders to tighten relevant bounds as the auction progresses. Further, iMLCA includes an entirely new third phase that forces further narrowing of the bounds to guarantee convergence (i.e., ensuring that bounds are tight enough such that the efficient allocation at reports can be determined). The overall iMLCA mechanism is provided in Algorithm 1. Before describing it in detail in the following subsections, we provide a high-level overview:

1. The initialization phase (Line 1; described in Section 4.1) queries random bundles to generate preliminary input for the ML algorithm.

2. The ML-based elicitation and bound refinement phase (Lines 2-8; described in Section 4.2) is the primary iterative elicitation phase of the mechanism. In this phase, the auctioneer repeatedly issues interval queries to the bidders via the ML-powered query module. Additionally, bidders are asked to tighten their bounds via the MRPAR activity rule, which drives revelation based on a provisional allocation and approximate linear clearing prices for the reported values. In each round of this phase, both the provisional allocation and approximate clearing prices are provided to the bidders; a maximum of $Q^{\max }$ rounds (e.g., 500) will occur.

3. The Convergence Phase (Lines 10-16; described in Section 4.3) continues iterative bound refinement to guarantee convergence, even when no linear clearing prices exist at the reported bids. Specifically, bidders are iteratively asked to tighten bounds according to the Interval Reduction activity rule, which is parameterized by an $\varepsilon$ tolerance that is stated to the bidders.

4. The outcome determination phase (Lines 17-18; described in Section 4.4) computes the final allocation and payments.

We now describe each of the four phases in detail.

\subsection{Initialization}

In this phase, each bidder is queried $Q^{\text {init }}$ (e.g., 50) bundles selected uniformly at random from the complete bundle space. In contrast to MLCA, the bidders answer these queries using upper 
and lower bounds. As in MLCA, the resulting reports are used as initial training data for the ML algorithm. We do not impose activity rules in the initialization phase; thus, bidders could initially report wide intervals, but at the cost of being forced to refine more dramatically in later rounds.

\subsection{Machine Learning-based Elicitation and Bound Refinement}

In this phase, the bidders are iteratively asked in rounds to both provide new bounds on additional bundles, and to refine their bounds on previously reported bundles. While notionally simple, there are several important details in realizing this design. Before diving into the details, we provide an overview of the four steps we will go through.

First, we will describe how queries for new bundles are generated, using an ML-based technique from Brero et al. (2021b), but modified to use interval queries (Section 4.2.1). Next, we will describe the MRPAR rule which is used to force bidders to narrow their bounds in each round (Section 4.2.2). The MRPAR rule requires bidders to respond to a provisional allocation, the determination of which we describe next (Section 4.2.3). Finally, we will introduce a new price-based activity rule that is better at driving elicitation than previous rules proposed in the literature (Section 4.2.4).

4.2.1. Generating new Queries iMLCA seeks to add bundles for both the main and the marginal economies (Lines 4 and 5 of Algorithm 1 respectively). In both economies, iMLCA requires bidders to respond to queries in each round, and it uses ML to inform which bundles to query. The actual selection of bundles via ML is achieved via the $M L$-powered query module of MLCA (Brero et al. 2021b, Algorithm 3), but in iMLCA, these queries are interval queries instead of the standard value queries used in MLCA. Switching for interval queries means that the ML algorithm used in the query module must generalize from bounds on each data point in its training data, rather than exact values. iMLCA works with any ML algorithm capable of doing this.

We adopt support vector regressions (SVRs) as our ML algorithm, as they can be modified to use bounds instead of exact values in their training data. Specifically, the standard $\varepsilon$-insensitive hinge loss function used in SVRs only penalizes the learner for predictions more than $\varepsilon$ away from the exact training data. It is mathematically straightforward to replace this $2 \varepsilon$-wide insensitivity region from the standard formulation with a data point-specific region capturing the bounds (see Brero et al. (2018)). Thus, in our usage, the SVR is only penalized for predictions outside of $[\underline{v}, \bar{v}]$. 
4.2.2. Bound Refinement In every round, iMLCA not only asks bidders about new bundles but also asks them to tighten their bounds on already-queried bundles $R_{i}$, according to an activity rule that balances elicitation against bidder effort (Algorithm 1, Line 6). Tighter bounds serve two purposes: first, they aid the ML algorithm in making better predictions, which improves the selection of new bundles to query. Second, given the price-based method used to drive them, they facilitate progress towards finding the most efficient allocation among those bundles that have been queried. We next provide details on this latter aspect and the activity rule that enables it.

Under the Modified Revealed Preference Activity Rule (MRPAR) (Lubin et al. 2008) bidders are provided both their portion of a provisional allocation $a_{i}^{\alpha}$ (Procedure 1 below), and a set of prices $\pi$ (Procedure 2 below). As the allocation is provided by the auctioneer, its feasibility can be enforced. Bidders must then make clear that the provisional bundle is their weakly preferred bundle at the quoted prices, or alternatively that some other bundle is strictly preferred. Formally, we have:

Activity Rule 1. Modified Revealed Preference Activity Rule (MRPAR) (Lubin ET AL. 2008):

Given a provisional allocation $a^{\alpha}$, linear prices $\pi$, and existing reports $R_{i}$, each bidder $i$ must submit reports revealing his preferred bundle $x_{i}^{*}$ by satisfying the following:

$$
\exists x_{i}^{*} \in R_{i} \text { s.t. } \underline{v}_{i}\left(x_{i}^{*}\right)-\pi\left(x_{i}^{*}\right) \geq \bar{v}_{i}\left(x_{i}\right)-\pi\left(x_{i}\right) \forall x_{i} \in R_{i} \backslash\left\{x_{i}^{*}\right\}
$$

where the inequality in Equation (2) must be strict if $x_{i}^{*} \neq a_{i}^{\alpha}$.

Activity Rule 1 sits within a class of activity rules that employ the principle of revealed preference (see Ausubel and Baranov (2017) for details). In general, such rules work by forcing bidders to reveal their preferred bundle at quoted prices, and then the mechanism updates prices in a direction that helps supply meet demand. Through this movement, the mechanism will eventually obtain clearing prices and a corresponding allocation that form a competitive equilibrium. When this happens, a special case of the First Welfare Theorem (Mas-Colell et al. 1995, 16.C-D) provides that the allocation will be efficient. Exact linear clearing prices may not exist in a combinatorial setting (Bikhchandani et al. 2002); thus, iMLCA may have only approximate clearing prices to work with. Fortunately, Lubin et al. (2008, Lemma 1) have anticipated this and shown that under 
approximate clearing prices we still obtain approximate efficiency. ${ }^{3}$ This highlights the importance of finding good approximately clearing prices $\pi$, a topic to which we will return in Section 4.2.4.

While satisfying Equation 2 may seem complicated, Figure 2 illustrates that the intuition behind the rule, moving bounds to identify the most preferred bundle at given prices, is easily visualized. Practical implementations of iMLCA would include bidding interfaces that provide such a visualization as an aid to bidders. We emphasize that, when responding to Activity Rule 1, bidders must only consider the small subset of bundles under consideration $R_{i}$, not the set of all possible bundles.

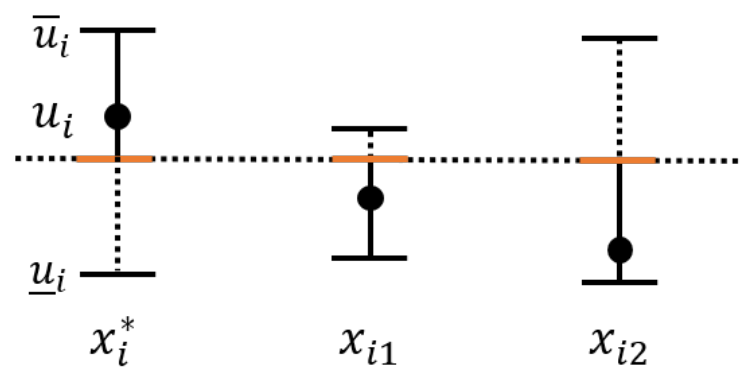

Figure 2: Illustration of Activity Rule 1. We see the scenario before refinement in black. The upper bars are the upper bound utility $\bar{u}_{i}(x)=$ $\bar{v}_{i}(x)-\pi(x)$, the lower bars are the lower bound utility $\underline{u}=\underline{v}_{i}(x)-\pi(x)$ and the dots are the true utility $\bar{u}_{i}(x)$ induced by prices $\pi$. A bidder must refine his bounds such that the lower bound utility on his preferred bundle $x^{*}$ is at the same level as the upper bound utility on all his other bundles (illustrated in orange).

4.2.3. Generating a Provisional Allocation To instantiate Activity Rule 1, we must first select a provisional allocation. Following Lubin et al. (2008), we choose the allocation by identifying a value profile that is an affine combination of the existing upper and lower bounds.

Procedure 1. Generate Provisional Allocation (Lubin et al. 2008)

For a chosen $\alpha \in[0,1]$, we compute a provisional valuation

$$
v_{i}^{\alpha}(x)=\alpha \underline{v}_{i}(x)+(1-\alpha) \bar{v}_{i}(x) \quad \forall i \in N, x \in R_{i}
$$

and a provisional allocation

$$
a^{\alpha} \in \underset{a \in \mathcal{F}}{\arg \max } \sum_{i \in N} v_{i}^{\alpha}\left(a_{i}\right)
$$

As in Lubin et al. (2008), we set $\alpha=\max \left\{0.5, \omega_{R}\right\}$, where $\omega_{R}$ is a convergence bound we will introduce in Section 4.3. As iMLCA proceeds, the provisional allocation moves towards the lower bounds (which are the ones ultimately implemented).

\footnotetext{
${ }^{3}$ The lemma is proven in a context where bidders state their full valuation function using a compact bidding language. By contrast, in our setting, the quoted efficiency is only with respect to the bundles under consideration, $R$.
} 
4.2.4. Generating Linear Prices Activity Rule 1 requires linear prices. Ideally, these would be clearing prices with respect to the reported values. However, in a CA, linear clearing prices may not exist. We therefore seek $\delta$-approximate linear clearing prices $\pi$ (at the given reports).

Definition 2 ( $\delta$-Approximate Clearing Prices At Reports). Given reported valuation profile $\hat{v}$ and an allocation $a$, prices $\pi$ are $\delta$-approximate clearing prices at reports if $\delta \geq \delta_{i k} \forall i, k$,

$$
\begin{aligned}
& \hat{v}_{i}\left(a_{i}\right)-\pi\left(a_{i}\right)+\delta_{i k} \geq \hat{v}_{i}\left(x_{i k}\right)-\pi\left(x_{i k}\right) \forall i, k, \quad \text { and } \\
& \pi_{j}=0 \quad \forall j \in M: \nexists i \in N: a_{i j}=1 .
\end{aligned}
$$

Equation (5) ensures that the clearing condition is violated by no more than $\delta_{i k}$ for any reported bundle $x_{i k}$, and $\delta$ is defined to bound all such $\delta_{i k}$. Equation (6) sets the price of any un-allocated item to zero, which ensures that the supply condition for clearing prices is met. Because it may easily be that $\delta_{i k} \neq \delta$ for many $k$, many price vectors may meet Definition 2 . We first break ties in favor of minimizing the number of positive $\delta_{i k}$ (see Procedure 4 in EC.A). We then break ties further, by selecting a price vector that reduces the number of bundles a bidder must consider in Activity Rule 1 (as we will show formally in Section 5). This procedure is as follows:

\section{Procedure 2. Generate PRICES}

i. Generate $\delta$-approximate clearing prices at reports with respect to the provisional valuation $v^{\alpha}$ and allocation $a^{\alpha}$; let $\delta_{i k}^{\alpha}$ be the required approximation (see Procedure 4 in EC.A).

ii. Minimize over all $\delta_{i k}^{\alpha}>0$ according to the objective $\left\|\delta_{i k}^{\alpha}\right\|_{2}$, while keeping constraints generated in step i.

iii. Generate $\delta$-approximate clearing prices at reports with respect to the provisional allocation $a^{\alpha}$ and the perturbed valuation $\stackrel{\bullet}{v}$ (defined with respect to $a^{\alpha}$ ) while keeping all previously generated constraints; let $\stackrel{\circ}{\delta}_{i k}$ be the required approximation.

iv. Minimize $\left\|\delta_{i k}+C\right\|_{2}$, holding all constraints active. Here $C>\max _{i, k}-\delta_{i k}$ is a constant added to ensure that negative $\delta_{i k}$ are properly ordered.

v. Solve a new program where the objective in step iv. becomes a constraint and we maximize the sum of the prices.

\subsection{Convergence}

When the second phase is complete, the bidders will have been guided to report and to refine bounds on bundles that are important for determining efficiency (via Activity Rule 1). However, we may not have elicited enough information to determine the efficient allocation at reports: Activity Rule 1's 
guarantee of convergence is approximate in the $\delta$ of the $\delta$-approximate linear clearing prices. We therefore continue into the third phase, where the Interval Reduction activity rule ensures bidders further refine the bounds on $Q^{\text {round }}$ already reported bundles (four, in our implementation), such that iMLCA can eventually identify the bundles belonging to an efficient outcome.

We use Algorithm 2 to identify those bundles where refinement is needed. The algorithm is careful to only include bundles where bounds are not yet tight enough (Lines 3 and 8). The identification of the bundles is based on a convergence bound $\omega_{R}=\underline{v}(\underline{a}) / \stackrel{\circ}{v}(\stackrel{\circ}{a}) \leq 1$; when $\omega_{R}=1$ convergence is achieved and the efficient allocation at reports can be guaranteed (Lubin et al. 2008, Equation 26). ${ }^{4}$ Based on $\omega_{R}$, tighter bounds on either $\underline{a}$ or $\stackrel{\circ}{a}$ will achieve progress towards convergence. Thus, Algorithm 2 identifies bundles in these allocations. However, a given bidder may already have been queried these bundles. When this happens, each such bidder $i$ is instead given the bundle for the next-best allocation $\stackrel{\circ}{a} \in \arg \max _{a \in \mathcal{F}^{\prime}} \stackrel{\circ}{v}(a)$, where allocations containing already queried bundles for bidder $i$ are excluded in $\mathcal{F}^{\prime}$ (Line 6 and 7). Algorithm 2 is run for each bidder $i$, producing $Q_{i}$, and these bundles then drive elicitation according to the following activity rule:

\footnotetext{
${ }^{4}$ Recall that the perturbed valuation $\stackrel{\circ}{v}$ can be used to measure the maximal surplus of choosing any bundle $x_{i}$ over $a_{i}$. Accordingly, the allocation with maximal such surplus is $\stackrel{\circ}{a} \in \arg \max _{a \in \mathcal{F}_{R}} \stackrel{\circ}{v}(a \mid \underline{a})$.
}

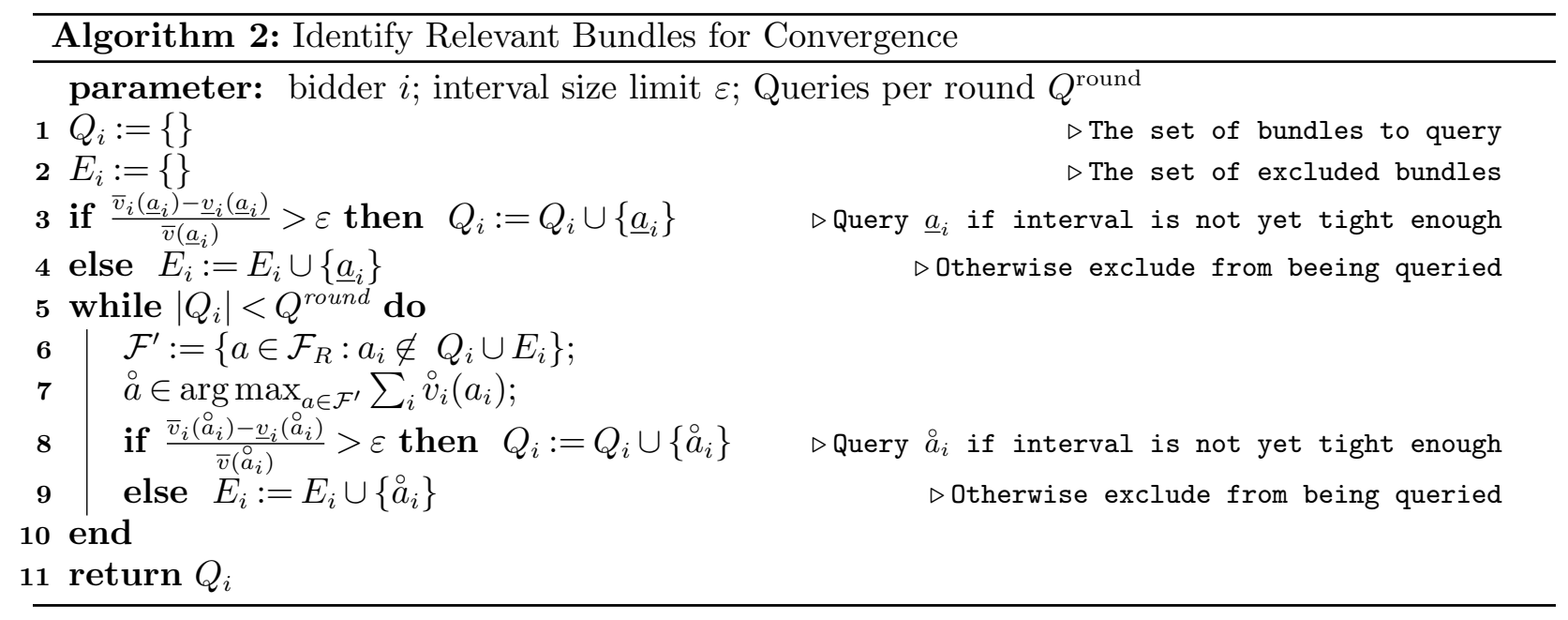




\section{Activity Rule 2. Interval Reduction Activity Rule}

Given $\varepsilon$ and bundle set $Q_{i}$, bidder $i$ must submit reports such that $\frac{\bar{v}_{i}(x)-\underline{v}_{i}(x)}{\bar{v}_{i}(x)} \leq \varepsilon \forall x \in Q_{i}$.

The rule forces revelation according to a relative measure (i.e. $\frac{\bar{v}(x)-\underline{v}(x)}{\bar{v}(x)}$, the ratio of interval size and the upper bound value). The rule is parameterized by $\varepsilon$, which specifies how much tightening is required. In each round, $\varepsilon$ is reduced by $50 \%$ in Algorithm 1 . Therefore, $\varepsilon \rightarrow 0$ in the number of rounds which guarantees convergence in the limit, i.e. tight bounds on $\underline{a}$ and $\stackrel{\circ}{a} .{ }^{5}$

For the Convergence Phase to terminate, we require the convergence bound to be achieved (i.e. $\omega^{R}=1$ ), guaranteeing we know the efficient allocation at reports. As we will discuss in Section 5.3, the size of the reported intervals for the final allocation pertains to the incentives of the mechanism and it is thus desirable for the auctioneer to have control over the gap size $(\bar{v}(\underline{a})-\underline{v}(\underline{a}))$ in the activity rule. To provide this control, the Convergence Phase only stops when the relative reported interval $\frac{\bar{v}(\underline{a})-\underline{v}(\underline{a})}{\bar{v}(\underline{a})}$ is no larger than $\varepsilon^{\text {stop }}$, which will be enforced by the Activity Rule 1 directly. We capture these two requirements in the stopping condition used by Algorithm 1, as follows:

Procedure 3. Convergence Stopping Rule

$\frac{\underline{v}\left(\frac{a}{\partial}\right)}{\hat{v}(a)}=1$ and $\frac{\bar{v}(\underline{a})-\underline{v}(\underline{a})}{\bar{v}(\underline{a})} \leq \varepsilon^{\text {stop }}$

\subsection{Outcome Determination}

iMLCA determines the final allocation based on the set of reported bundles only; thus, the set of feasible allocations is $\mathcal{F}_{R}=\left\{a \in \mathcal{F}: a_{i} \in R_{i} \forall i\right\}$. The final allocation $\underline{a}$ is determined by $\arg \max _{a \in \mathcal{F}_{R}} \sum_{i \in N} \underline{v}_{i}\left(a_{i}\right)$. When determining the allocation, we take a conservative approach by using the lower bound valuation $\underline{v}$ (Line 17 of Algorithm 1). This guarantees that we never implement an outcome that is higher than a bidder's true value (under truthful bidding), which ensures individual rationality. Finally, we also use $\underline{v}$ to compute VCG-style payments (Line 18 of Algorithm 1). We discuss the incentive implications of this design in Section 5.3.

\footnotetext{
${ }^{5}$ In our experiments (Section 6.2) we do not reach the limit of $\varepsilon=0$, instead stopping according to Procedure 3.
} 


\subsection{Additional Design Features of iMLCA}

Similarly to Brero et al. (2021b), we equip iMLCA with three additional design features that will likely be important in many domains. First, we allow bidders to provide additional information to the mechanism by reporting unsolicited bundle-value pairs in the initialization phase; we refer to these as "push bids." Second, we introduce a parameter $Q^{\text {round }}$ that determines the number of queries the mechanism asks each bidder in each round of the second phase of the auction; this parameter provides the auctioneer with additional control over the number of rounds. Last, we allow the auctioneer to consider alternative payment rules to VCG. One of them is the VCG-nearest rule (Day and Cramton 2012), which we also test in our experiments.

\section{Theoretical Analysis}

In this section, we present a theoretical analysis of iMLCA. We begin by analyzing standard mechanism design properties, specifically individual rationality (Subsection 5.1), no-deficit (Subsection 5.2), and bidders' incentives (Subsection 5.3). Next we analyze the implication of our new price-based activity rule on bidder effort (Subsection 5.4). Finally, we discuss bounding the efficiency loss in the performance of the ML algorithm (Remark 1).

\subsection{Individual Rationality}

A mechanism satisfies individual rationality if no bidder pays more than his reported value.

Proposition 1 (Individual Rationality). iMLCA satisfies individual rationality.

The proof is provided in EC.B.

\subsection{No-Deficit}

A mechanism satisfies no-deficit if the sum of all payments is weakly positive. We have:

Proposition 2 (No-Deficit). iMLCA satisfies no deficit.

The proof is provided in EC.C.

\subsection{Incentives}

In this section, we discuss the incentives of iMLCA. Like all spectrum auction designs used in practice (e.g., CCA; Ausubel and Baranov 2017), iMLCA is not strategyproof. This is not surprising, 
given that also MLCA (using exact value reports) is susceptible to certain manipulations (Brero et al. 2021b). However, we can offer a parallel argument to that of Brero et al. (2021b) to argue that, in practice, iMLCA provides bidders with good incentives to report truthfully. We start by defining a truthful strategy for bidders reporting upper and lower bounds.

Definition 3 (Truthful Strategy in iMLCA). In iMLCA, a bidder's strategy is called truthful if the bidder only reports upper and lower bounds that are consistent with his true values.

Because iMLCA charges VCG payments, the utility of each bidder $i$ can be formulated as follows:

$$
u_{i}=\underbrace{v\left(\underline{a}_{i}\right)+\sum_{j \neq i} \underline{v}_{j}\left(\underline{a}_{j}\right)}_{\text {(I) welfare main economy }}-\underbrace{\sum_{j \neq i} \underline{v}_{j}\left(a_{j}^{-i}\right)}_{\text {(II) welfare marginal economy }}
$$

Term (I) is the sum of $i$ 's true value and the reported lower bound of the other bidders for $\underline{a}$, and (II) is the reported lower bound of the other bidders for $a^{-i}$. If bidder $i$ wants to increase his utility, he must increase the difference (I) - (II).

We distinguish two pathways for manipulations in iMLCA:

1. Bidder $i$ tries to change the allocation in his main or marginal economy.

2. Bidder $i$ tries to affect the tightness of other bidders' bounds in his main or marginal economy.

The first pathway already existed in MLCA. The second pathway is new in iMLCA. We now explain why iMLCA is robust against both manipulation pathways in practice.

Changing the allocation in the main or marginal economy. A bidder may try to decrease term (II) by affecting which allocation the mechanism eventually uses for his marginal economy. However, following the same argument as for MLCA, we note that (II) is practically independent of bidder $i$ 's report because iMLCA explicitly generates queries for each bidder's marginal economy (i.e., excluding all previous reports by bidder $i$ ). Given this, the only remaining way for bidder $i$ to improve his utility via an allocation change is to change the final allocation $\underline{a}$ towards an allocation that has higher social welfare. But as in MLCA, iMLCA allows bidders to submit "push bids" in the initialization phase, enabling them to push information to the auction that they deem useful. 
Thus, if they have a bundle in mind which they believe will increase social welfare, they can simply submit a corresponding push bid. For more details, please see Brero et al. (2021b).

Affecting the tightness of other bidders' bounds. In iMLCA, even without affecting an allocation change, a bidder can try to increase his utility by (a) inducing bidders to tighten their bounds as much as possible on the final allocation $\underline{a}$ (i.e., increasing term (I)), or by (b) inducing bidders to keep their bounds in his marginal economy as loose as possible (i.e., reducing term (II)). iMLCA explicitly addresses (a) as follows: In the Convergence Phase, the auctioneer can control the size of the reported intervals for the final allocation directly by choosing the parameter $\varepsilon^{\text {stop }}$. If $\varepsilon^{\text {stop }}$ is small enough, a bidder cannot force tighter bounds and thus iMLCA prevents such manipulations by design. ${ }^{6}$ Regarding (b), we note that iMLCA only requests bound refinements for bundles relevant for the final allocation $\underline{a}$. Thus, as there is no explicit bound refinement process in the marginals, any remaining manipulation strategies seem implausible to execute in practice.

By making the following assumptions, we can derive more formal incentive guarantees:

Assumption 1. For every bidder $i$, if all other bidders report truthfully, then the reported welfare of bidder i's marginal economy is independent of bidder i's value reports.

As we have discussed above, explicitly querying each bidder $i$ 's marginal economy makes $a^{-i}$ practically independent of his reports, supporting (but not guaranteeing) Assumption 1.

Assumption 2. When all bidders are truthful, all bidders' lower bounds for the final allocation reported at the end of the auction, are equal to the true values, i.e., for each bidder $i: \underline{v}_{i}\left(a_{i}\right)=v_{i}\left(a_{i}\right)$.

By setting a sufficiently low $\varepsilon^{\text {stop }}$, the auctioneer can guarantee that, for each bidder $i$, the equation $\underline{v}_{i}\left(a_{i}\right)=v_{i}\left(a_{i}\right)$ gets arbitrarily close to holding with equality, supporting Assumption 2 . Assumptions 1 and 2 allow us to extend the social welfare alignment property of MLCA to iMLCA: Proposition 3 (Social Welfare Alignment). If Assumptions 1 and 2 hold and all other bidders are truthful, then a bidder can only increase his utility by increasing the reported social welfare of $\underline{a}$.

\footnotetext{
${ }^{6}$ In fact, an auctioneer can balance information revelation (i.e., tighter intervals) and incentive properties by choosing $\varepsilon^{\text {stop }}$. However, $\varepsilon^{\text {stop }}$ only affects a small subset of reports and the intervals of all other reports can remain loose.
} 
Proof. The proof follows in a straightforward way by considering Equation (7). Because of Assumption 1, we know that each bidder cannot affect the reported welfare in his marginal economy. From Assumption 2, and because bidders are truthful, we have that the term (I) in Equation (7) corresponds to the social welfare in the allocation $\underline{a}$. Thus, the only way for a bidder to increase his utility is by increasing the reported social welfare for the final allocation $\underline{a}$.

When combined with the opportunity to submit push bids, social welfare alignment already provides strong incentives for truthful reporting. Concretely, instead of trying to game the mechanism to lead it to a more efficient allocation, a bidder can push truthful information he has about promising bundles to the auctioneer. That said, social welfare alignment is not sufficient to guarantee that a bidder cannot accidentally drive the mechanism to a better allocation via manipulations, without having this allocation in mind. To address this last scenario and obtain a formal equilibrium property, we need one more assumption:

Assumption 3. When all bidders are truthful then the final allocation of iMLCA is efficient.

As efficiency can be bounded in the quality of the ML algorithm (see Remark 1 below), the assumption relies on the quality of the ML-algorithm. Using SVRs, Assumption 3 holds in the majority of auction instances for two of the domains we study (see Section 6.2), but not for the third one. If Assumption 3 indeed holds, then we obtain the following result.

Proposition 4 (Truthful Reporting is an Ex-post Nash Equilibrium). If Assumptions 1, 2, and 3 hold, truthful reporting is an ex post Nash equilibrium of the game induced by iMLCA.

Proof. The proof follows by considering that, under Assumptions 1 and 2, iMLCA is social welfare aligned. Using Assumption 3, we have that this social welfare (and thus bidder $i$ 's utility) is maximized when bidder $i$ is truthful. This concludes the proof.

\subsection{Bidder Effort Reduction}


When a bidder is asked to refine his reports according to Activity Rule 1, there may be many bundles that need not be considered at all. Specifically, bidder $i$ need not consider any bundle $x_{i}$ for which the utility interval induced by the given prices $\pi$ does not overlap with the utility interval of the provisionally allocated bundle. This effect is illustrated in Figure 3. The following proposition shows that all bundles for which $\stackrel{\circ}{\delta}_{i k} \leq$ 0 need not be considered according to Activity Rule 1.

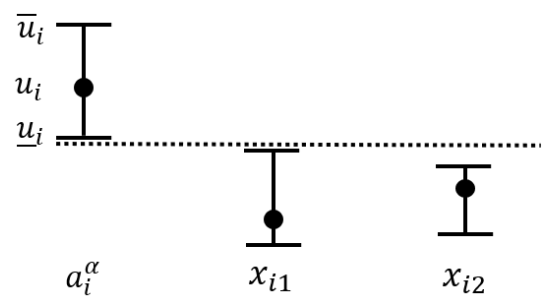

Figure 3: Illustration of bundles that need not be considered by a bidder $i$ when responding to Activity Rule 1. Shown is the scenario prior to refinement for given prices $\pi$. The bidder need not consider $x_{i 1}$ and $x_{i 2}$ as it is immediate from the location of the bounds that neither can be his preferred bundle.

Proposition 5 (Bidder Effort). Bidder $i$ can ignore all bundles $x_{i k} \in R_{i} \backslash\left\{a_{i}^{\alpha}\right\}$ with $\stackrel{\circ}{i k}_{i k} 0$ when responding to Activity Rule 1.

The proof is provided in EC.D.

As we maximize the number of such $\stackrel{\circ}{i k}_{i k}$ in Step iii. of Procedure 2, we are guaranteed to reduce the bidder effort in terms of bundles to consider during an Activity Rule 1 refinement, when compared to alternative price generation procedures that omit this step (e.g., that of Kwasnica et al. 2005). Our new linear price selection algorithm is helping us achieve our goal of reducing bidder elicitation effort; it may also be of use in other revealed preference-based mechanisms.

Remark 1 (Bounding the Efficiency Loss). We can bound the efficiency loss of the mechanism in the prediction performance of the ML-algorithm, following a similar result for MLCA (Brero et al. 2018). Surprisingly, even though iMLCA only has interval information to work with, it can reason about those intervals sufficiently well that the bound on efficiency is no weaker than that for MLCA. The full proposition and proof are deferred to EC.E in the interest of space.

\section{Experimental Evaluation}

In this section, we evaluate the performance of iMLCA by comparing it against the original MLCA and the CCA. We consider MLCA to be an upper bound benchmark for iMLCA, given that MLCA is similar in design but works with more precise information. 


\subsection{Experiment Set-Up}

6.1.1. Domains. For our experiments, we use the spectrum auction test suite (SATS) version 0.8.0. (Weiss et al. 2017). SATS enables us to generate thousands of synthetic auction instances on demand, providing access to bidders' values, and allowing us to quickly compute the true efficient allocation. We use the following three domains:

Global Synergy Value Model (GSVM) (Goeree and Holt 2010): A relatively simple medium-sized domain with 18 items and 7 bidders of different types.

Local Synergy Value Model (LSVM) (Scheffel et al. 2012): A medium-sized domain with 18 items and 6 bidders of different types. Synergies arise from the spatial proximity of items, which makes LSVM more complex.

Multi-Region Value Model (MRVM) (Weiss et al. 2017): A large-sized domain with 98 items and 10 bidders of different types. It models the complex 700Mhz Canadian spectrum auction.

6.1.2. Modeling Bidder Behavior. When simulating bidders' responses to queries in our experiments, we let bidders behave consistently with their true preferences. Thus, for every bidder $i$ and every bundle $x \in R_{i}$, it holds that: $\underline{v}_{i}(x) \leq v_{i}(x) \leq \bar{v}_{i}(x)$. As we do not want the reported intervals $\left[\underline{v}_{i}(x), \bar{v}_{i}(x)\right]$ to always be centered around the true value $v_{i}(x)$, we simulate the interval reports following the same approach as Brero et al. (2018): For any given bundle $x$ and bidder $i$, we independently draw two error measures $z_{1}$ and $z_{2}$ from a standard normal distribution with mean 0 and standard deviation $\mu$, where $\mu$ is a parameter capturing the bidder's reported interval size. The lower and upper bounds are then obtained as $\underline{v}_{i}(x)=\max \left(0, v_{i}(x)\left(1-z_{1}\right)\right)$ and $\bar{v}_{i}(x)=v_{i}(x)\left(1+z_{2}\right)$, respectively. In our experiments, we use $\mu=0.5$. To provide some intuition: with $\mu=0.5$, a typical interval report is $[100,200]$. To simulate the process by which bidders tighten their bounds, we have implemented two straightforward heuristics that adjust bounds to meet Activity Rule 1 and Activity Rule 1 respectively. For details, please see EC.F. 


\begin{tabular}{rcrrrr} 
Domain & Mechanism & Efficiency & Revenue & Revenue (Core) & Rounds \\
\hline \hline \multirow{3}{*}{ GSVM } & iMLCA & $100.0 \%(0.01)$ & $58.5 \%(1.32)$ & $63.1 \%(1.22)$ & $16(0.1)$ \\
\cline { 2 - 6 } & MLCA & $100.0 \%(0.00)$ & $68.4 \%(1.10)$ & $72.4 \%(1.05)$ & $14(0.0)$ \\
\cline { 2 - 6 } & CCA & $100.0 \%(0.00)$ & $68.1 \%(1.13)$ & $73.1 \%(0.99)$ & $235(3.0)$ \\
\hline \hline \multirow{3}{*}{ LSVM } & iMLCA & $99.6 \%(0.10)$ & $74.4 \%(1.22)$ & $78.7 \%(1.12)$ & $122(1.2)$ \\
\cline { 2 - 6 } & MLCA & $99.6 \%(0.12)$ & $80.9 \%(0.91)$ & $84.5 \%(0.83)$ & $114(0.0)$ \\
\cline { 2 - 6 } MRVM & CCA & $99.9 \%(0.03)$ & $82.3 \%(0.91)$ & $86.4 \%(0.73)$ & $125(0.3)$ \\
\cline { 2 - 6 } & iMLCA & $96.2 \%(0.15)$ & $23.8 \%(0.66)$ & $25.1 \%(0.57)$ & $122(0.4)$ \\
\cline { 2 - 6 } & MLCA & $96.4 \%(0.13)$ & $40.5 \%(0.33)$ & $40.7 \%(0.34)$ & $114(0.0)$ \\
\cline { 2 - 6 } & CCA & $94.2 \%(0.20)$ & $30.0 \%(0.77)$ & $34.5 \%(0.39)$ & $142(0.9)$
\end{tabular}

Table 1 Results for iMLCA, MLCA and CCA with a maximal number of reports of 500 (100 for GSVM). Standard errors in parentheses. Averages over 100 instances (50 for MRVM).

6.1.3. Mechanism Set-Up. For iMLCA, we use the same parameters as were used by Brero et al. (2021b) for $\mathrm{MLCA}^{7}$. We set the final interval size limit $\varepsilon^{\text {stop }}=0.005$. For example, this corresponds to a maximal interval of the reported social welfare of $[99.5,100]$. The refinement parameter $\alpha$ is obtained as described in Lubin et al. (2008). To solve all optimization problems, we use CPLEX (version 12.10) and adopt a time limit for some problems (including the new price generation problem) similar to Brero et al. (2021b). ${ }^{8}$ Our source code is publicly available under an open-source license at https://github.com/marketdesignresearch/mechlib.

\subsection{Results: Comparing iMLCA, MLCA and CCA}

Table 1 provides a comparison of iMLCA, MLCA and CCA in all three domains. ${ }^{9}$ The efficiency of an allocation $a$ is measured as $v(a) / v\left(a^{*}\right)$ and revenue as the total payment relative to the maximal social welfare $\sum_{i} p_{i} / v\left(a^{*}\right)$. We further report alternative VCG-nearest core selecting payments (Day and Cramton 2012) and the total number of rounds (including all phases of iMLCA).

We focus our analysis on efficiency, as spectrum auctions are generally government-run auctions that target efficiency and not revenue maximization (Cramton 2013). We use a one-way ANOVA

${ }^{7}$ The parameters adopted from Brero et al. (2021b) include the SVR parametrization, $Q^{\text {init }}=50, Q^{\text {max }}=500\left(Q^{\text {max }}=\right.$ 100 for GSVM) and the number of interval queries per round and bidder in the ML-based elicitation and bound refinement phase $Q^{\text {round }}$ is set to 4 . The same $Q^{\text {round }}$ is used as parameter for Algorithm 2.

${ }^{8}$ We used an Ubuntu 16.04 cluster with AMD EPYC $77022.0 \mathrm{GHz}$ processors with 8 cores and 32 GB of RAM. We use the best sub-optimal solution found when the time limit is reached.

${ }^{9}$ To enable a head-to-head comparison, we use the same setting seeds (for the test set) as used by Brero et al. (2021b) which are seeds 101 - 200 for GSVM and LSVM, and seeds 101 - 150 for MRVM. 
to test for statistically significant differences between all three mechanisms, and we use a post-hoc Tukey test for further pairwise comparisons.

In GSVM, all three mechanisms perform extremely well with $100.0 \%$ efficiency (with no statistically significant difference; $p>0.1$. Next, we consider LSVM, which is more complex and thus more difficult to learn with the kernel-based SVRs we employ (see Brero et al. 2021b). Here, we find a statistically significant difference between the three mechanisms $(p<0.05)$. Further analysis shows that the CCA performs better than MLCA $(p<0.05)$. However, CCA and iMLCA do not perform statistically significantly different $(p=0.08)$. Furthermore, MLCA and iMLCA do not perform statistically significantly different $(p=0.84)$

To put the results into perspective, we note that GSVM and LSVM are very simple test domains. In fact, the regional bidders in GSVM have value for less than 64 bundles. Thus, the CCA can elicit those bidders' full value functions, because they can report their value for up to 100 bundles in the supplementary round. This gives the CCA a natural advantage in these simple domains compared to MLCA and iMLCA, which do not have information about the bidders' bundles of interest and must therefore content with the full bundle space. In light of this, it is particularly impressive that iMLCA does not perform worse than the CCA in GSVM nor in LSVM.

Next, we consider MRVM, where we find a statistically significant difference between the three mechanisms $(p<0.001)$. When we compare the mechanisms pairwise, we find no statistically significant difference between the efficiency of MLCA and iMLCA $(p=0.65)$. By contrast, the efficiency of the CCA is lower than the efficiency of iMLCA $(p=0.001)$ and of MLCA $(p=0.001)$.

Remark 2. Our experimental results also show that iMLCA achieves slightly lower revenue than the other mechanisms. This is due to our activity rules driving more elicitation in the main rather than the marginal economies. We discuss potential ways to increase iMLCA's revenue in Section 7.

Summarizing our main experiments, we conclude that iMLCA achieves the same efficiency as MLCA, even though it operates with interval queries instead of exact queries. Furthermore, iMLCA achieves a $2 \%$ point higher efficiency than the CCA in the realistically-sized MRVM domain. 


\begin{tabular}{|c|c|c|c|c|c|c|}
\hline Mechanism & Domain & $\begin{array}{c}\text { Initial } \\
\text { Interval } \\
\text { Size }\end{array}$ & $\begin{array}{l}\text { Final } \\
\text { Interval } \\
\text { Size }\end{array}$ & Domain & $\begin{array}{c}\text { True } \\
\text { Manipulability } \\
\text { Bound }\end{array}$ & $\begin{array}{l}\text { Observable } \\
\text { Manipulability } \\
\text { Bound }\end{array}$ \\
\hline \multirow{3}{*}{ iMLCA } & GSVM & $55 \%$ & $46 \%$ & GSVM & $0.1 \%(0.01)$ & $0.2 \%(0.01)$ \\
\hline & LSVM & $55 \%$ & $48 \%$ & LSVM & $0.0 \%(0.00)$ & $0.0 \%(0.01)$ \\
\hline & MRVM & $55 \%$ & $41 \%$ & MRVM & $0.0 \%(0.00)$ & $0.0 \%(0.01)$ \\
\hline MLCA & all domains & $0 \%$ & $0 \%$ & \multirow{2}{*}{\multicolumn{3}{|c|}{$\begin{array}{l}\text { Table } 3 \text { The true manipulability bound reports the } \\
\text { relative difference of the lower bound and true value. } \\
\text { The observable manipulability bound reports the } \\
\text { relative difference of the lower and upper bound. Both } \\
\text { measures total over the final allocation. Standard errors } \\
\text { in parentheses. Mean of } 100 \text { instances ( } 50 \text { for MRVM). }\end{array}$}} \\
\hline $\begin{array}{l}\text { Table } 2 \\
\text { interval siz } \\
\text { itialization p } \\
\text { of }\end{array}$ & \multicolumn{3}{|c|}{$\begin{array}{l}\text { Comparison of average reported relative } \\
\text { ize between iMLCA and MLCA after the } \\
\text { phase and at the end of the auction. Mean } \\
\text { f } 100 \text { instances ( } 50 \text { for MRVM). }\end{array}$} & & & \\
\hline
\end{tabular}

\section{3. $\quad$ Reducing Bidder Effort}

In this section, we show that iMLCA achieves a reduction in bidder effort over MLCA, which was our main design goal and which is iMLCA's main advantage.

6.3.1. Evaluating Reported Interval Size As our measure for bidder effort, we introduce the relative size of all reported intervals. Concretely, for a report $\left(x_{i k}, \underline{v}_{i k}, \bar{v}_{i k}\right)$ we measure the relative interval size as $\left(\bar{v}_{i k}-\underline{v}_{i k}\right) / \bar{v}_{i k}$. In Table 2 , we compare MLCA and iMLCA in terms of the initial and final interval size (averaged over the reported bundles) for all bundles with a positive upper bound value (i.e., all bundles a bidder is interested in). For MLCA, the interval is always 0, because bidders must report exact values. In contrast, for iMLCA, the final interval size is between $46 \%$ and $41 \%$. Note that even $41 \%$ corresponds to remarkably loose intervals. To see this, consider that with an average final interval size of $41 \%$, a typical interval is [59,100]. In EC.G, we also report the distribution over these interval sizes. These results highlight that iMLCA allows bidders to maintain large intervals on the majority of their reported values despite iteratively asking bidders to refine their bounds and forcing relatively tight intervals for the final allocation.

6.3.2. $\quad$ Effect of New Convergence Phase Next, we evaluate the effectiveness of our novel Convergence Phase. To do this, we compare it against a version of iMLCA where the Convergence Phase is replaced by the DIAR activity rule as proposed by (Lubin et al. 2008); we report these results in EC.H. In summary, the results show that across all domains, the DIAR-based mechanism needs at least 100 rounds more to achieve convergence. Furthermore, the final interval sizes for the DIAR-based mechanism are tighter than in iMLCA, meaning that bidders need to reveal more information when using DIAR. 
6.3.3. Effect of New Price Generation Objective Finally, we analyze by how much our new price-based refinement process (Procedure 2) improves the performance of iMLCA, which we report in EC.J. There we observe that Procedure 2 reduces bidder effort in terms of number of bundles a bidder must consider during one Activity Rule 1 refinement $(p<0.001)$ and in terms of the total number of reports refined per bidder $(p<0.001)$. We also see that iMLCA achieves a higher efficiency under these prices relative to earlier pricing approaches $(p<0.01)$.

\subsection{Bidder Incentives}

Next, we provide experimental evidence about the incentive properties of our mechanism.

\subsubsection{Interval Reduction Activity Rule As discussed in Section 5.3, to prevent manipu-} lations in the main economy, it is important that the reported lower bounds for the final allocation are close to the true values. While the auctioneer has no knowledge of the size of this interval, she can control the observed interval size (i.e., the difference between upper and lower bounds) via the parameter $\varepsilon^{\text {stop }}$. We report both intervals in Table 3. The relative true manipulability bound reports the tightness of the lower bound with respect to the true value, i.e. $v(\underline{a})-\underline{v}(\underline{a})) / v(\underline{a})$, while the relative observable manipulability bound is $\bar{v}(\underline{a})-\underline{v}(\underline{a})) / \bar{v}(\underline{a})$. We see that, in all domains, the true manipulability bound is $\leq 0.1 \%$, which means that the mechanism leaves no practically meaningful opportunities for manipulating the bounds of the final allocation.

6.4.2. Bound Manipulation Experiments Because bidders specify intervals in iMLCA, there is a new potential manipulation path that was not present in MLCA: bidders strategically manipulating their bounds. To support our incentive analysis (see Section 5.3), we ran experiments to explore this potential manipulation. In these experiments, we hold all bidders' bound-reporting strategies fixed (as described in Section 6), other than a single deviating bidder. We then evaluate each of the following bound generation heuristics for this deviating bidder: Exact Values, where the focal bidder reports exact values; Standard Bounds, where the focal bidder uses the standard bound-reporting strategy where the random noise in the initial bounds is created with a standard 
deviation of 0.5; Wider Bounds, where the deviating bidder instead uses a standard deviation of 1; and Maximum Bounds, where the deviating bidder reports our numerical equivalent to $[0, \infty]$.

For space considerations, the details of these strategies and corresponding results are deferred to EC.I. The key take-away is that, when running a one-way ANOVA over bidder utility, we observe a p-value that is $>0.637$ in all cases and over all domains. Thus, we find no evidence that bidders can improve their utility by reporting narrower or wider intervals.

\section{Discussion}

Our experimental evidence provides strong support for the efficacy of iMLCA, achieving the same efficiency as MLCA but with lower bidder effort. Specifically, using linear prices to drive refinement over a restricted set of bundles in iMLCA seems as effective as the exact value queries in MLCA.

Our experimental results on the manipulability of iMLCA require care in interpretation. These experiments do not evaluate the efficacy of non-truthful behavior in equilibrium, but rather the efficacy of a unilateral deviation from our baseline strategy. We believe that these experiments are a valuable second-best, given that a full equilibrium analysis of an ICA is currently out of reach.

In our bound manipulation experiments, we have only studied bidders strategically reporting narrower or wider bounds, but we have not simultaneously considered letting bidders report intervals that imply a value misreport (e.g., considering the set of manipulation strategies analyzed by Brero et al. (2021b)). Studying the cross-product of value misreporting strategies and bound manipulation strategies is computationally very challenging, but would be interesting future work.

In our experiments, iMLCA exhibits lower revenue than MLCA and the CCA. This is not surprising, given that our activity rules are designed to drive more elicitation in the main economy than in the marginal economies. Thus, the reported bounds in the marginal economy allocations may remain looser, resulting in smaller VCG payments (Line 18 of Algorithm 1). For the spectrum auctions that motivate this work it is efficiency, not revenue, that is the design goal. But for other settings, revenue could be important. Thus, in future work, the activity rules could be altered to seek additional refinement in the marginal economies, which would raise revenue. 


\section{Conclusion}

In this paper, we have developed a new ML-powered ICA with interval bidding (iMLCA), which avoids the high costs associated with reporting exact values. Our experiments have demonstrated that iMLCA achieves the same efficiency as MLCA, and even outperforms the CCA in the realistically-sized MRVM domain. This suggests iMLCA as a practical mechanism to conduct large ICAs. We emphasize that our goal was not to beat MLCA, but rather to offer comparable performance while requiring less information from the bidders (intervals instead of exact values).

On a technical level, our main contribution was the careful integration of a price-based refinement process with an ML-powered query module in one auction phase. To address incentives, we highlight our new Convergence Phase that enables the auctioneer to make a deliberate trade-off between elicitation effort and robustness to manipulation.

We have used one specific measure of bidder effort, namely the relative size of the reported intervals. One could also consider other measures (e.g., from the behavioral economics literature), including non-linear measures capturing that narrowing already small bounds may be harder than narrowing loose bounds. One interesting question for future work would be to investigate how iMLCA could be modified to directly target such non-linear bidder effort measures. It would be interesting to complement these efforts with lab experiments, studying how bidders perceive iMLCA compared to other auction designs.

Another exciting direction for future work is to study how iMLCA could be modified to work in domains without money. For example, for the course allocation problem, Budish and Kessler (2021) have recently shown that the A-CEEI mechanism (which uses a notion of clearing prices in a virtual currency), while much better than earlier designs, still does not allow all students to accurately and consistently report their preferences. It would be interesting to study whether iterative designs like iMLCA could ameliorate these issues.

\section{Acknowledgments}

Part of this research was supported by the European Research Council (ERC) under the European Unions Horizon 2020 research and innovation programme (Grant agreement No. 805542). This material is based upon work supported by the National Science Foundation under grant no. CMMI-1761163. 


\section{References}

Ausubel L, Cramton P (2011) Auction design for wind rights. Technical report, Report to Bureau of Ocean Energy Management, Regulation and Enforcement.

Ausubel LM, Baranov O (2017) A practical guide to the combinatorial clock auction. The Economic Journal 127(605):334-350.

Beyeler M, Brero G, Lubin B, Seuken S (2021) Machine learning-powered iterative combinatorial auctions with interval bidding. Proceedings of the 22nd ACM Conference on Economics and Computation, 136.

Bichler M, Paulsen P (2018) A principal-agent model of bidding firms in multi-unit auctions. Games and Economic Behavior 111:20-40.

Bichler M, Shabalin P, Wolf J (2013) Do core-selecting combinatorial clock auctions always lead to high efficiency? An experimental analysis of spectrum auction designs. Experimental Economics 16(4):511545.

Bikhchandani S, Ostroy JM, et al. (2002) The package assignment model. Journal of Economic theory 107(2):377-406.

Blum A, Jackson J, Sandholm T, Zinkevich M (2004) Preference elicitation and query learning. Journal of Machine Learning Research 5(Jun):649-667.

Brero G, Eden A, Gerstgrasser M, Parkes DC, Rheingans-Yoo D (2021a) Reinforcement learning of simple indirect mechanisms. Proceedings of the 35th AAAI Conference of Artificial Intelligence (Virtual).

Brero G, Lahaie S (2018) A bayesian clearing mechanism for combinatorial auctions. Proceedings of the 32nd AAAI Conference on Artificial Intelligence, 941-948.

Brero G, Lahaie S, Seuken S (2019) Fast iterative combinatorial auctions via bayesian learning. Proceedings of the 33rd AAAI Conference on Artificial Intelligence, 1820-1828.

Brero G, Lubin B, Seuken S (2018) Combinatorial Auctions via Machine Learning-based Preference Elicitation. Proceedings of the 27th International Joint Conference on Artificial Intelligence, 128-136.

Brero G, Lubin B, Seuken S (2021b) Machine learning-powered iterative combinatorial auctions. arXiv preprint abs/1911.08042. 
Budish E, Kessler JB (2021) Can market participants report their preferences accurately (enough)? Management Science, Forthcoming.

Bünz B, Lubin B, Seuken S (2018) Designing core-selecting payment rules: A computational search approach. Proceedings of the 19th ACM Conference on Economics and Computation (Ithaca, NY).

Cavallo R, Parkes DC, Juda AI, Kirsch A, Kulesza A, Lahaie S, Lubin B, Michael L, Shneidman J (2005) Tbbl: A tree-based bidding language for iterative combinatorial exchanges. Multidisciplinary Workshop on Advances in Preference Handling.

Cramton P (2013) Spectrum auction design. Review of industrial organization 42(2):161-190.

Day RW, Cramton P (2012) Quadratic core-selecting payment rules for combinatorial auctions. Operations Research 60(3):588-603.

Dütting P, Feng Z, Narasimhan H, Parkes D, Ravindranath SS (2019) Optimal auctions through deep learning. International Conference on Machine Learning, 1706-1715 (PMLR).

Dütting P, Fischer F, Jirapinyo P, Lai JK, Lubin B, Parkes DC (2015) Payment rules through discriminantbased classifiers. ACM Transactions on Economics and Computation (TEAC) 3(1):1-41.

Garey M, Johnson DS (1978) "Strong" np-completeness results: Motivation, examples, and implications. Journal of the ACM 25:499-508.

Goeree JK, Holt CA (2010) Hierarchical package bidding: A paper \& pencil combinatorial auction. Games and Economic Behavior 70(1):146-169.

Goetzendorf A, Bichler M, Shabalin P, Day RW (2015) Compact Bid Languages and Core Pricing in Large Multi-item Auctions. Management Science 61(7):1684-1703.

Golowich N, Narasimhan H, Parkes DC (2018) Deep learning for multi-facility location mechanism design. Proceedings of the 27th International Joint Conference on Artificial Intelligence, 261-267.

Kwasnica AM, Ledyard JO, Porter D, DeMartini C (2005) A new and improved design for multiobject iterative auctions. Management science 51(3):419-434.

Lahaie SM, Parkes DC (2004) Applying learning algorithms to preference elicitation. Proceedings of the 5th ACM conference on Electronic commerce, 180-188. 
Lubin B, Juda AI, Cavallo R, Lahaie S, Shneidman J, Parkes DC (2008) Ice: An expressive iterative combinatorial exchange. Journal of Artificial Intelligence Research 33:33-77.

Mas-Colell A, Whinston MD, Green JR, et al. (1995) Microeconomic theory, volume 1 (Oxford university press New York).

Oren ME, Williams AC (1975) On competitive bidding. Operations Research 23(6):1072-1079.

Parkes DC (2006) Iterative combinatorial auctions. Cramton P, Shoham Y, Steinberg R, eds., Combinatorial Auctions, chapter 2, 41-78 (MIT Press).

Sandholm T (2013) Very-large-scale generalized combinatorial multi-attribute auctions: Lessons from conducting $\$ 60$ billion of sourcing. Vulkan N, Roth AE, Neeman Z, eds., The Handbook of Market Design, chapter 1, 379-412 (Oxford, UK: Oxford University Press).

Sandholm T, Boutilier C (2006) Preference Elicitation in Combinatorial Auctions. Cramton P, Shoham Y, Steinberg R, eds., Combinatorial Auctions, chapter 10 (The MIT Press).

Scheffel T, Ziegler G, Bichler M (2012) On the impact of package selection in combinatorial auctions: an experimental study in the context of spectrum auction design. Experimental Economics 15(4):667-692.

Shen W, Lahaie S, Leme RP (2019) Learning to clear the market. Proceedings of the 36th International Conference on Machine Learning, 5710-5718 (Longbeach, CA).

Tang P (2017) Reinforcement mechanism design. Proceedings of the 26th International Joint Conference on Artificial Intelligence (Melbourne, Australia).

Weiss M, Lubin B, Seuken S (2017) SATS: A Universal Spectrum Auction Test Suite. Proceedings of the Sixteenth Conference on Autonomous Agents and MultiAgent Systems, 51-59 (Sao Paulo, Brazil).

Weissteiner J, Seuken S (2020) Deep learning-powered iterative combinatorial auctions. Proceedings of the Thirty-fourth AAAI Conference on Artificial Intelligence, 2284-2293 (New York, NY).

Weissteiner J, Wendler C, Seuken S, Lubin B, Püschel M (2021) Fourier analysis-based iterative combinatorial auctions. arxiv preprint abs/2009.10749. 


\section{Electronic Companion for: \\ iMLCA: Machine Learning-powered Iterative Combinatorial Auctions with Interval Bidding}

\author{
Benjamin Lubin ${ }^{\dagger}$ \\ Boston University \\ blubin@bu.edu
}

\author{
Sven Seuken ${ }^{\dagger}$ \\ University of Zurich \\ seuken@ifi.uzh.ch
}

\author{
Manuel Beyeler ${ }^{\dagger}$ \\ University of Zurich \\ manuel.beyeler@uzh.ch
}

Gianluca Brero

Harvard University

gbrero@g.harvard.edu

\section{EC.A: Compute $\delta$-approximate Clearing Prices at reports}

Procedure 4. Compute $\delta$-Approximate Clearing Prices at Reports

Solve a sequence of linear programs to find $\pi$. First:

$$
\begin{aligned}
& \delta=\min _{i, k} \max _{i k} \\
& \text { s.t. } \hat{v}_{i}\left(a_{i}^{\alpha}\right)-\pi\left(a_{i}^{\alpha}\right)+\delta_{i k} \geq \hat{v}_{i}\left(x_{i k}\right)-\pi\left(x_{i k}\right) \forall i, k \\
& \quad \pi_{j}=0 \quad \forall j \in M: \nexists i \in N: a_{i j}^{\alpha}=1
\end{aligned}
$$

Where $\delta_{i k}$ measures violation of the clearing condition for the $k$ th report of bidder $i$. Thus, the overall maximal negative utility of any bidder given prices $\pi$ is $\delta$. Next solve:

$$
\begin{gathered}
\min \left|\left\{\delta_{i k}: \delta_{i k}>0\right\}\right| \\
\text { s.t. }(9) \text { and }(10) \\
\delta_{i k} \leq \delta \quad \forall i, k
\end{gathered}
$$

which breaks ties to miminize the number of positive $\delta_{i k}$.

\section{EC.B: Proof: Individual Rationality}

Proof. The reported lower bound utility of each bidder $i$ is non-negative as $\underline{v}_{i}(\underline{a})-p_{i}=\underline{v}(\underline{a})-\sum_{j \neq i} \underline{v}_{j}\left(a_{j}^{-i}\right) \geq$ 0. Indeed, the marginal allocation $a_{j}^{-i}$ is also a feasible allocation for the main economy (i.e $a_{j}^{-i} \in \mathcal{F}_{R}$ ) but the allocation with the highest lower bound value in the main economy is $\underline{a} \in \arg \max _{a \in \mathcal{F}_{R}} \underline{v}(a)$. Therefore we have $\underline{v}(\underline{a}) \geq \sum_{j \neq i} \underline{v}_{j}\left(a_{j}^{-i}\right)$.

\section{EC.C: Proof: No-Deficit}

Proof. The payment of each bidder $i$ is non-negative, i.e., $p_{i}=\sum_{j \neq i}\left(\underline{v}_{j}\left(a_{j}^{-i}\right)-\underline{v}_{j}\left(\underline{a}_{j}\right)\right) \geq 0$. This is the case because $a_{j}^{-i} \in \mathcal{F}_{R}$ and $a_{j}^{-i} \in \mathcal{F}_{R}$ are both feasible allocations with respect to all reports $R$, but $a_{j}^{-i} \in$ $\arg \max _{a \in \mathcal{F}_{R}} \sum_{j \neq i} \underline{v}_{i}(a)$ is the allocation that maximizes the lowerbound value for the marginal economy.

Thus, we have $\sum_{j \neq i} \underline{v}_{j}\left(a_{j}^{-i}\right) \geq \sum_{j \neq i} \underline{v}_{j}\left(\underline{a}_{j}\right)$.

\footnotetext{
$\dagger$ These authors have contributed equally to this paper.
} 


\section{EC.D: Proof: Bidder Effort}

Proof. Before refinement, it holds that $\underline{v}_{i}\left(a_{i}^{\alpha}\right)-\pi\left(a_{i}^{\alpha}\right) \geq \bar{v}_{i}\left(x_{i k}\right)-\pi\left(x_{i k}\right)$ for all bundles $x_{i k} \neq a_{i}^{\alpha}$ with $\stackrel{\circ}{\delta}_{i k} \leq 0$. But according to Activity Rule 1 bidder $i$ must report some $x_{i}^{*} \neq x_{i k}$ such that $\underline{v}_{i}\left(x_{i}^{*}\right)-\pi\left(x_{i}^{*}\right) \geq \underline{v}\left(a_{i}^{\alpha}\right)-$ $\pi\left(a_{i}^{\alpha}\right) \geq \bar{v}_{i}\left(x_{i k}\right)-\pi\left(x_{i k}\right)$.

\section{EC.E: Bounding Efficiency Loss in ML Performance}

We can bound the efficiency loss of the mechanism in the prediction performance of the ML-algorithm, following a similar result for MLCA (Brero et al. 2018). Surprisingly, even though iMLCA only has interval information to work with, it can reason about those intervals sufficiently well that the bound on efficiency is no weaker than that for MLCA, as provided in the following proposition:

Proposition 6 (Efficiency Bound). Assume that bidders' interval reports are consistent with their true values. Let $\tilde{v}$ be some valuation profile learned by the $M L$ algorithm, let $\tilde{a}$ be an efficient allocation w.r.t. to $\tilde{v}$, and let $a^{*}$ be an efficient allocation w.r.t. the true valuation profile. Assume that the learning errors in $\tilde{a}$ and $a^{*}$ are bounded as follows: for each bidder $i,\left|\tilde{v}_{i}(\tilde{a})-v_{i}(\tilde{a})\right| \leq \delta_{1}$ and $\left|\tilde{v}_{i}\left(a^{*}\right)-v_{i}\left(a^{*}\right)\right| \leq \delta_{2}$, for $\delta_{1}, \delta_{2} \in \mathbb{R}$. Then the following bound on the efficiency loss in the final allocation a holds for all $\tilde{v}$ learned for any economy, in any iteration of $i M L C A$ :

$$
1-\frac{v(\underline{a})}{v\left(a^{*}\right)} \leq \frac{n\left(\delta_{1}+\delta_{2}\right)}{v\left(a^{*}\right)}
$$

Proof. Let $a^{+} \in \max _{a \in F_{R}} v(a)$ be the allocation that maximized the welfare with respect to reports and assuming truthful reports we have according to Brero et al. (2021b)

$$
\frac{v\left(a^{+}\right)}{v\left(a^{*}\right)} \geq 1-\frac{n\left(\delta_{1}+\delta_{2}\right)}{v\left(a^{*}\right)}
$$

and according to Lubin et al. (2008)

$$
\frac{v(\underline{a})}{v\left(a^{+}\right)} \geq \frac{\underline{v}(\underline{a})}{\dot{v}(\stackrel{\circ}{a})}
$$

Multiplying these inequalities and from the fact that $\frac{v(a)}{v\left(\frac{a}{a}\right)}=1$ at the end of iMLCA due the stopping condition of the Convergence Phase (Procedure 3) the proposition follows.

\section{EC.F: Modeling Bidder Behavior - Refinement}

We implemented two straightforward heuristics that adjust bounds according to Activity Rule 1 and Activity Rule 1, respectively We first run the MRPAR heuristic (Algorithm 3) followed by the Interval Reduction 
activity rule heuristic (Algorithm 4). The MRPAR heuristic relies on the assumption that it might be hard for a bidder to determine his exact value and therefore a bidder rather chooses to tighten bounds that are further away from his true value. In both heuristics, we aim to minimize the amount of refinement, such that bidders only refine the bounds as much as is needed to comply with the activity rules.
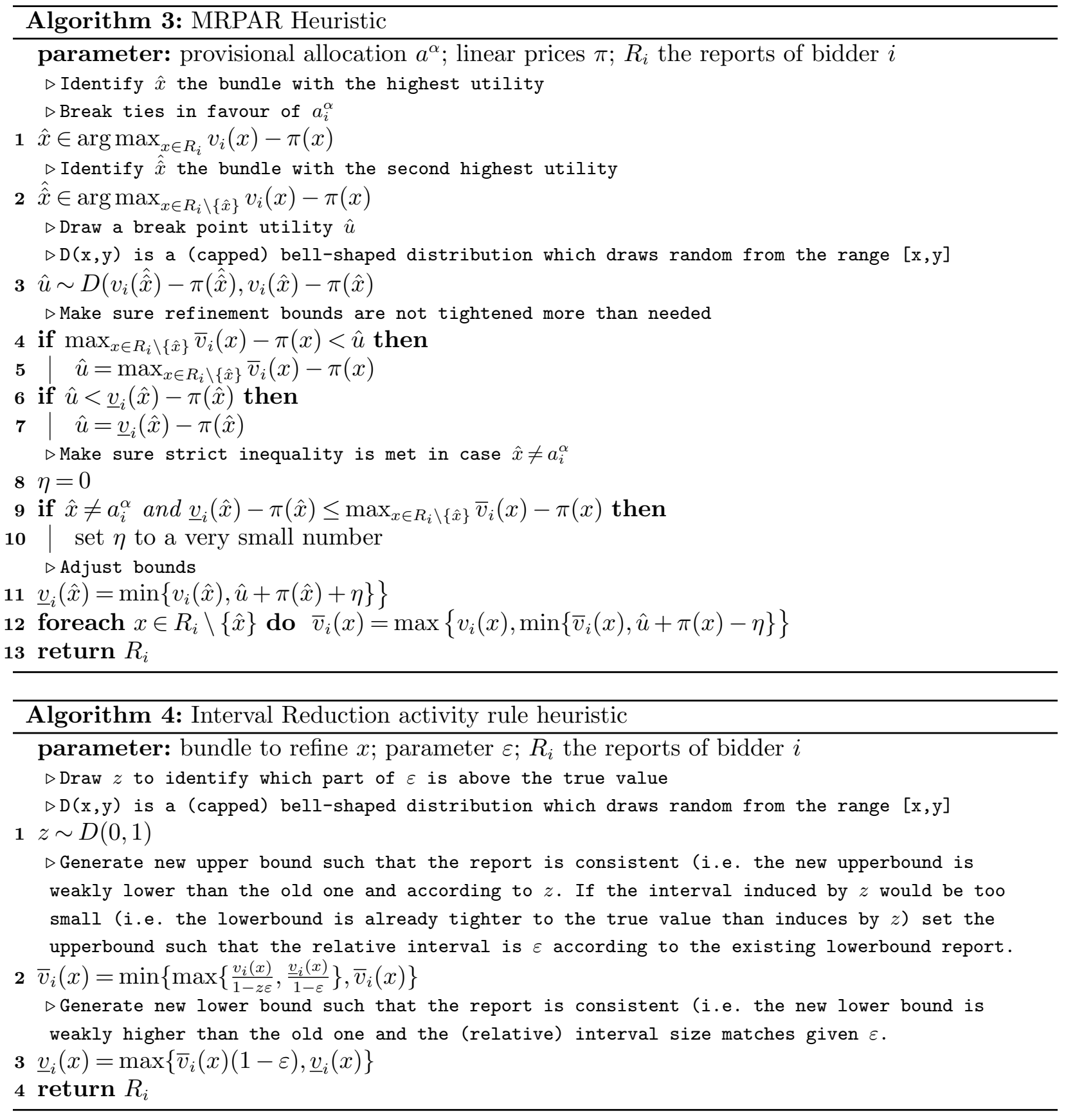

\section{EC.G: Distribution of Interval Size}

In Figure 4 we provide a comparison of the interval size distribution between the initial and final reports in the MRVM domain. We see that while the reported intervals of the final implemented allocation are almost 
tight, most others remain loose. Specifically, at the end of the auction only $19 \%$ of the reports have an interval size of $0 \%$ to $10 \%$ while $37 \%$ of the reports have a interval size of more than $50 \%$.

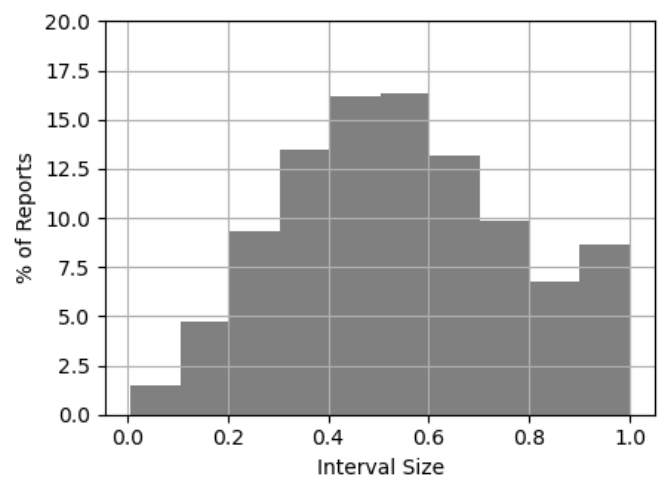

(a) Initial Interval Size

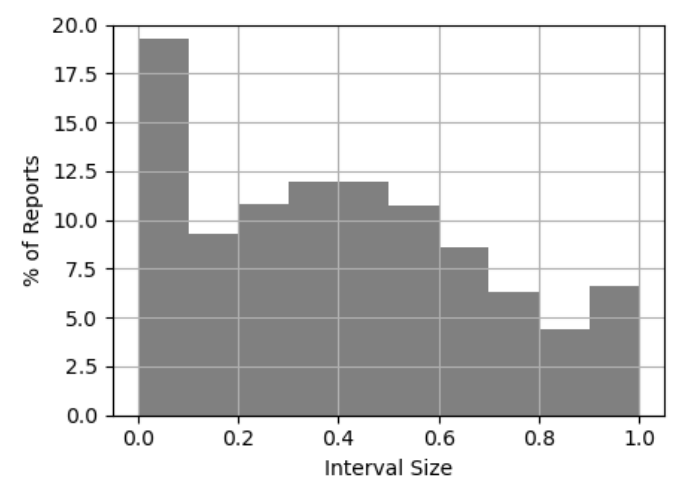

(b) Final Inverval Size

Figure 4 Comparison of the reported interval size distribution after the initialization phase and at the end of the auction.

\section{EC.H: Evaluation of the Convergence Phase}

To evaluate the effectiveness of our novel Convergence Phase, we compare our rule with a version of iMLCA where the Convergence Phase is replaced by the DIAR activity rule as proposed by (Lubin et al. 2008). We refer to this version as iMLCA-DIAR. iMLCA-DIAR stops the refinement when $\frac{v(a)}{v(a)}=1$, i.e. the allocation that is guaranteed to maximizes reported values is identified. The results are shown in table 4 . We see clearly that iMLCA-DIAR needs at least 100 rounds more to achieve convergence than iMLCA with the new Convergence Phase. Furthermore final interval sizes for iMLCA-DIAR are tighter than in iMLCa, i.e. bidder needs to reveal more information in iMLCA-diar. Note that the difference in efficiency in MRVM between iMLCA and iMLCA-DIAR is caused by some randomness in the ML-based Elicitation and Bound Refinement Phase (i.e. CPLEX timeouts) and not due to the different final phase.

Domain Mechanism Efficiency Revenue Revenue (Core) Rounds Final Interval

Size

\begin{tabular}{lcrrrrr}
\hline \hline \multirow{2}{*}{ GSVM } & iMLCA & $100.0 \%(0.01)$ & $58.5 \%(1.32)$ & $63.1 \%(1.22)$ & $16(0.1)$ & $46 \%$ \\
\cline { 2 - 7 } & iMLCA-diar & $100.0 \%(0.01)$ & $59.7 \%(1.42)$ & $64.4 \%(1.30)$ & $135(20.4)$ & $34 \%$ \\
\hline \hline \multirow{2}{*}{ LSVM } & iMLCA & $99.6 \%(0.10)$ & $74.4 \%(1.22)$ & $78.7 \%(1.12)$ & $122(1.2)$ & $48 \%$ \\
\cline { 2 - 6 } & iMLCA-diar & $99.6 \%(0.10)$ & $74.6 \%(1.20)$ & $79.1 \%(1.10)$ & $317(29.9)$ & $39 \%$ \\
\hline \hline \multirow{2}{*}{ MRVM } & iMLCA & $96.2 \%(0.15)$ & $23.8 \%(0.66)$ & $25.1 \%(0.57)$ & $122(0.4)$ & $41 \%$ \\
\cline { 2 - 7 } & iMLCA-diar & $96.1 \%(0.14)$ & $30.7 \%(1.22)$ & $31.4 \%(1.17)$ & $496(59.5)$ & $33 \%$ \\
\hline
\end{tabular}

Table 4 Results for iMLCA and iMLCA-DIAR with a maximal number of reports of 500 (100 for GSVM). Standard errors in parentheses. Averages over 100 instances (50 for MRVM). 


\section{EC.I: Manipulation Experiments}

To support our incentive analysis (see section 5.3), we ran experiments to explore if a bidder can improve his utility by strategically reporting his bounds. In these experiments we hold all bidders' bound-reporting approach fixed at our standard procedure (as described in 6), other than a single deviating bidder. We then evaluate each of the following bound generation heuristics for this deviating bidder: ${ }^{1}$

Exact Values $(S D=0)$ The deviating bidder reports exact values, i.e. for each report for bundle $x$ we have $\underline{v}(x)=\bar{v}(x)=v(x)$.

Standard Bounds $(S D=0.5)$ The bidder simply uses the standard approach, as described in Section 6. Here, SD refers to the standard deviation used when drawing the noise used to generate bounds and we use our baseline level of $S D=0.5$.

Wider Bounds $(S D=1)$ The deviating bidder generates bounds where the interval size is in expectation doubled (i.e. each initial report for bundle $x$ we have $\bar{v}(x)-v(x)$ and $v(x)-\underline{v}(x)$ is in expectation twice as large as in the standard bound generation procedure.

Maximum Bounds (0,Inf) The deviating bidders initial bounds for each bundle is supposed to be $[0, \infty]$. For our simulation we use 1.5 times social welfare to represent $\infty$ for numerical reasons.

The results are shown in Table 5 for GSVM, Table 6 for LSVM, and Table 7 for MRVM. For each domain we tested deviations for all bidder types and report the resulting social welfare at truth, the marginal economy welfare for the deviating bidder at reported lower bounds and the utility of the deviating bidder. ${ }^{2}$

We observe very little difference in social welfare, marginal economy welfare or utility under any of the deviating strategies. To formalize this, in the last row we show the p-value of the one-way ANOVA to test for statistically significant differences across all applied strategies for each column respectively.

Focusing on utility, we observe very high p-values (in all cases $>0.637$ ) for the utility in all domains and therefore can conclude that it is very unlikely that a bidder can improve his utility by using these strategies. A similar argument follows for social welfare and marginal economy welfare.

${ }^{1}$ All of these heuristics are 'safe' in that they the bidder's true value always lies within his reported bounds

${ }^{2}$ The prices are computed based on reported lower bounds such that the utility cannot exactly be decomposed from the other two reported measures. 


\begin{tabular}{|c|c|c|c|c|c|c|}
\hline & \multicolumn{3}{|c|}{ Regional } & \multicolumn{3}{|c|}{ National } \\
\hline & $\begin{array}{l}\text { Social } \\
\text { Welfare }\end{array}$ & $\begin{array}{l}\text { Margin } \\
\text { Econor } \\
\text { Welfar } \\
\text { (at Lo } \\
\text { Bound }\end{array}$ & Utility & $\begin{array}{l}\text { Social } \\
\text { Welfare }\end{array}$ & $\begin{array}{l}\text { Marginal } \\
\text { Economy } \\
\text { Welfare } \\
\text { (at Lower } \\
\text { Bounds) }\end{array}$ & Utility \\
\hline Standard Bounds $(\mathrm{SD}=0.5)$ & $\begin{array}{l}437.5 \\
(3.6)\end{array}$ & $\begin{array}{l}412.1 \\
(3.7)\end{array}$ & $\begin{array}{l}25.19 \\
(2.25)\end{array}$ & $\begin{array}{l}437.5 \\
(3.6)\end{array}$ & $\begin{array}{l}432.4 \\
(4.1)\end{array}$ & $\begin{array}{l}4.78 \\
(1.35)\end{array}$ \\
\hline Exact Values $(\mathrm{SD}=0)$ & $\begin{array}{l}437.5 \\
(3.6)\end{array}$ & $\begin{array}{l}411.8 \\
(3.7)\end{array}$ & $\begin{array}{l}25.48 \\
(2.29)\end{array}$ & $\begin{array}{l}437.5 \\
(3.6)\end{array}$ & $\begin{array}{l}431.7 \\
(4.2)\end{array}$ & $\begin{array}{l}5.47 \\
(1.62)\end{array}$ \\
\hline Wider Bounds $(\mathrm{SD}=1)$ & $\begin{array}{l}437.4 \\
(3.6)\end{array}$ & $\begin{array}{l}412.6 \\
(3.6)\end{array}$ & $\begin{array}{l}24.55 \\
(2.19)\end{array}$ & $\begin{array}{l}437.1 \\
(3.6)\end{array}$ & $\begin{array}{l}432.5 \\
(4.1)\end{array}$ & $\begin{array}{l}4.37 \\
(1.41)\end{array}$ \\
\hline Maximum Bounds (0, Inf) & $\begin{array}{l}437.4 \\
(3.6)\end{array}$ & $\begin{array}{l}414.6 \\
(3.6)\end{array}$ & $\begin{array}{l}22.56 \\
(2.07)\end{array}$ & $\begin{array}{l}436.5 \\
(3.7)\end{array}$ & $\begin{array}{l}432.6 \\
(4.1)\end{array}$ & $\begin{array}{l}3.65 \\
(1.35)\end{array}$ \\
\hline ANOVA p-value & 1.000 & 0.944 & 0.787 & 0.997 & 0.999 & 0.841 \\
\hline
\end{tabular}

Table 5 Results for different bound reporting strategies for one bidder of each reported type in GSVM. Standard errors in parentheses. Averages over 100 instances.

\begin{tabular}{|c|c|c|c|c|c|c|}
\hline & \multicolumn{3}{|c|}{ Regional } & \multicolumn{3}{|c|}{ National } \\
\hline & $\begin{array}{l}\text { Social } \\
\text { Welfare }\end{array}$ & $\begin{array}{l}\text { Margir } \\
\text { Econor } \\
\text { Welfar } \\
\text { (at Lo } \\
\text { Bound }\end{array}$ & Utility & $\begin{array}{l}\text { Social } \\
\text { Welfare }\end{array}$ & $\begin{array}{l}\text { Marginal } \\
\text { Economy } \\
\text { Welfare } \\
\text { (at Lowe } \\
\text { Bounds) }\end{array}$ & Utility \\
\hline Standard Bounds $(\mathrm{SD}=0.5)$ & $\begin{array}{l}533.5 \\
(4.5)\end{array}$ & $\begin{array}{l}520.6 \\
(6.0)\end{array}$ & $\begin{array}{l}12.88 \\
(2.93)\end{array}$ & $\begin{array}{l}533.5 \\
(4.5)\end{array}$ & $\begin{array}{l}505.4 \\
(4.6)\end{array}$ & $\begin{array}{l}28.04 \\
(3.89)\end{array}$ \\
\hline Exact Values $(\mathrm{SD}=0)$ & $\begin{array}{l}532.8 \\
(4.5)\end{array}$ & $\begin{array}{l}520.3 \\
(6.1)\end{array}$ & $\begin{array}{l}12.46 \\
(2.93)\end{array}$ & $\begin{array}{l}533.4 \\
(4.5)\end{array}$ & $\begin{array}{l}505.5 \\
(4.8)\end{array}$ & $\begin{array}{l}27.89 \\
(3.59)\end{array}$ \\
\hline Wider Bounds $(\mathrm{SD}=1)$ & $\begin{array}{l}534.1 \\
(4.4)\end{array}$ & $\begin{array}{l}520.4 \\
(6.1)\end{array}$ & $\begin{array}{l}13.67 \\
(3.32)\end{array}$ & $\begin{array}{l}533.7 \\
(4.4)\end{array}$ & $\begin{array}{l}507.2 \\
(4.6)\end{array}$ & $\begin{array}{l}26.46 \\
(3.66)\end{array}$ \\
\hline Maximum Bounds $(0$, Inf $)$ & $\begin{array}{l}533.2 \\
(4.5)\end{array}$ & $\begin{array}{l}520.9 \\
(6.0)\end{array}$ & $\begin{array}{l}12.35 \\
(2.93)\end{array}$ & $\begin{array}{l}533.0 \\
(4.5)\end{array}$ & $\begin{array}{l}510.1 \\
(4.3)\end{array}$ & $\begin{array}{l}22.81 \\
(3.19)\end{array}$ \\
\hline ANOVA p-value & 0.998 & 1.000 & 0.990 & 1.000 & 0.875 & 0.714 \\
\hline
\end{tabular}

Table 6 Results for different bound reporting strategies for one bidder of each reported type in LSVM. Standard errors in parentheses. Averages over 100 instances. 


\begin{tabular}{|c|c|c|c|c|c|c|c|c|c|}
\hline & \multicolumn{3}{|c|}{ Local } & \multicolumn{3}{|c|}{ Regional } & \multicolumn{3}{|c|}{ National } \\
\hline & $\begin{array}{l}\text { Socia } \\
\text { Wel- } \\
\text { fare }\end{array}$ & $\begin{array}{l}1 \text { Marginal } \\
\text { Economy } \\
\text { Welfare } \\
\text { (at Lower } \\
\text { Bounds) }\end{array}$ & Utility & $\begin{array}{l}\text { Socia } \\
\text { Wel- } \\
\text { fare }\end{array}$ & $\begin{array}{l}\text { Marginal } \\
\text { Economy } \\
\text { Welfare } \\
\text { (at Lower } \\
\text { Bounds) }\end{array}$ & Utility & $\begin{array}{l}\text { Socia } \\
\text { Wel- } \\
\text { fare }\end{array}$ & $\begin{array}{l}\text { Marginal } \\
\text { Economy } \\
\text { Welfare } \\
\text { (at Lower } \\
\text { Bounds) }\end{array}$ & Utility \\
\hline $\begin{array}{l}\text { Standard Bounds } \\
(\mathrm{SD}=0.5)\end{array}$ & $\begin{array}{l}9842 \\
(129)\end{array}$ & $\begin{array}{l}9841 \\
(129)\end{array}$ & $\begin{array}{l}0.09 \\
(0.08)\end{array}$ & $\begin{array}{l}9842 \\
(129)\end{array}$ & $\begin{array}{l}9822 \\
(128)\end{array}$ & $\begin{array}{l}18.63 \\
(5.27)\end{array}$ & $\begin{array}{l}9842 \\
(129)\end{array}$ & $\begin{array}{l}7468 \\
(152)\end{array}$ & $\begin{array}{l}2373 \\
(110)\end{array}$ \\
\hline $\begin{array}{ll}\text { Exact } & \text { Values } \\
(\mathrm{SD}=0) & \end{array}$ & $\begin{array}{l}9814 \\
(126)\end{array}$ & $\begin{array}{l}9813 \\
(126)\end{array}$ & $\begin{array}{l}0.04 \\
(0.03)\end{array}$ & $\begin{array}{l}9830 \\
(124)\end{array}$ & $\begin{array}{l}9806 \\
(123)\end{array}$ & $\begin{array}{l}21.91 \\
(6.30)\end{array}$ & $\begin{array}{l}9733 \\
(125)\end{array}$ & $\begin{array}{l}7384 \\
(156)\end{array}$ & $\begin{array}{l}2345 \\
(116)\end{array}$ \\
\hline $\begin{array}{l}\text { Wider } \quad \text { Bounds } \\
(\mathrm{SD}=1)\end{array}$ & $\begin{array}{l}9825 \\
(131)\end{array}$ & $\begin{array}{l}9824 \\
(131)\end{array}$ & $\begin{array}{l}0.08 \\
(0.08)\end{array}$ & $\begin{array}{l}9841 \\
(129)\end{array}$ & $\begin{array}{l}9821 \\
(128)\end{array}$ & $\begin{array}{l}18.71 \\
(5.60)\end{array}$ & $\begin{array}{l}9830 \\
(127)\end{array}$ & $\begin{array}{l}7441 \\
(158)\end{array}$ & $\begin{array}{l}2388 \\
(109)\end{array}$ \\
\hline $\begin{array}{l}\text { Maximum } \\
\text { Bounds }(0, \text { Inf })\end{array}$ & $\begin{array}{l}9814 \\
(127)\end{array}$ & $\begin{array}{l}9813 \\
(127)\end{array}$ & $\begin{array}{l}0.00 \\
(0.00)\end{array}$ & $\begin{array}{l}9804 \\
(129)\end{array}$ & $\begin{array}{l}9791 \\
(129)\end{array}$ & $\begin{array}{l}12.19 \\
(4.00)\end{array}$ & $\begin{array}{l}9815 \\
(127)\end{array}$ & $\begin{array}{l}7457 \\
(150)\end{array}$ & $\begin{array}{l}2357 \\
(104)\end{array}$ \\
\hline ANOVA p-value & 0.998 & 0.998 & 0.691 & 0.996 & 0.998 & 0.637 & 0.931 & 0.981 & 0.993 \\
\hline
\end{tabular}

Table 7 Results for different bound reporting strategies for one bidder of each reported type in MRVM. Values in Millions. Standard errors in parentheses. Averages over 100 instances.

\section{EC.J: Evaluation of Enhanced Price Generation Procedure}

Here, we consider the MRVM domain and compare iMLCA to a version of iMLCA where we use a simpler price generation procedure inspired by Kwasnica et al. (2005), which we refer to as iMLCA-K. The prices used in this alternative are $\delta$-approximate clearing prices with respect to $v^{\alpha}$ only. ${ }^{3}$ Table 8 shows that our new price generation objective reduces bidder effort in terms of number of bundles a bidder must consider during one Activity Rule 1 refinement $(p<0.001)$ and in terms of the total number of reports refined per bidder throughout the auction $(p<0.001)$. We also see that iMLCA achieves higher efficiency $(p<0.01)$ than iMLCA-K.

\begin{tabular}{lccccc} 
Mechanism & Efficiency & $\begin{array}{c}\text { \# MRPAR } \\
\text { Refinement }\end{array}$ & $\begin{array}{c}\text { Total \# of } \\
\text { Refinements }\end{array}$ & Rounds & $\begin{array}{c}\text { Final } \\
\text { Interval } \\
\text { Size }\end{array}$ \\
\hline iMLCA & $96.2 \%(0.15)$ & $26.4(0.51)$ & $970.8(14.05)$ & $122(0.4)$ & $41 \%$ \\
\hline iMLCA-K & $95.5 \%(0.16)$ & $35.4(0.29)$ & $1065.4(11.13)$ & $123(0.4)$ & $40 \%$
\end{tabular}

Table 8 Effect of iMLCAs' new price generation compared to a version of iMLCA using a price generation procedure inspired by Kwasnica et al. (2005) (iMLCA-K). Results for MRVM. Standard errors in parentheses. Averages over 50 instances.

\footnotetext{
${ }^{3}$ The price generation procedure proposed by Lubin et al. (2008) is also based on Kwasnica et al. (2005), while they apply different tie-breaking rules to approximate final payments.
} 